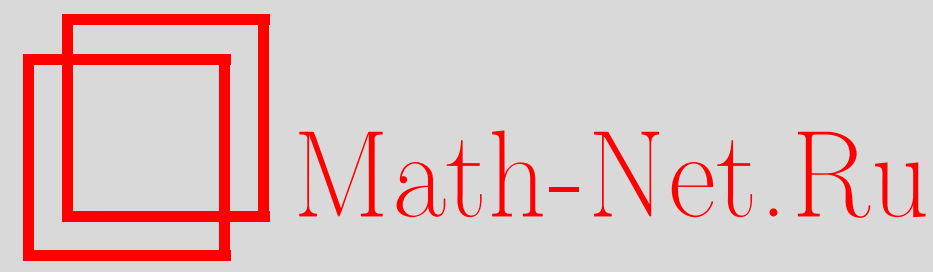

В. М. Харламов, Вик. С. Куликов, О брэйд-монодромных разложениях на множители, Изв. РАН. Сер. матем., 2003, том 67, выпуск 3, 79-118

DOI: https://doi.org/10.4213/im436

Использование Общероссийского математического портала Math-Net.Ru подразумевает, что вы прочитали и согласны с пользовательским соглашением

http://www . mathnet.ru/rus/agreement

Параметры загрузки:

IP: 18.207 .199 .55

26 апреля 2023 г., 14:54:25 
УДК 512.772.1+514.756.44

Вик. С. Куликов, В.М. Харламов

\title{
О брэйд-монодромных разложениях на множители
}

\author{
К 80-летию Игоря Ростиславовича Шафаревича
}

\begin{abstract}
Введено понятие полугруппы над группой кос и на языке полугрупп изучены разложения брэйд-монодромии на множители (bmf) плоских алгебраических кривых и некоторых других близких к ним объектов. В качестве одного из приложений приведено новое доказательство теоремы Оревкова о реализуемости любого брэйд-монодромного разложения на множители как bmf алгебраической кривой над диском и показано, что сложность такой реализации не может быть ограничена в терминах типов множителей разложения брэйд-монодромии. Кроме того, доказано, что типы брэйд-монодромного разложения на множители различают с точностью до $H$-изотопии кривые Гурвица с особыми точками нерасщепляемого типа и различают каспидальные $J$-голоморфные кривые в $\mathbb{C P}^{2}$ с точностью до симплектической изотопии.
\end{abstract}

Библиография: 23 наименования.

\section{Введение}

В настоящей статье мы имеем дело с алгебраическими кривыми и другими близкими к ним объектами, лежащими в проективной плоскости, такими как $J$-голоморфные кривые и кривые Гурвица (определение кривых Гурвица дано в $\S 3$ ), поведение которых относительно некоторого пучка прямых аналогично поведению плоских алгебраических кривых. Обшая природа всех этих геометрических объектов состоит в том, что для каждого из них может быть определено так называемое разложение брәйд-монодромии на множители (кратко bmf), являющееся мощным инструментом изучения топологии вложения алгебраических кривых в $\mathbb{C P}^{2}$. Начиная с основополагаюших работ О. Кизини [6], [7], разложения брэйдмонодромии рассматриваются как настояшие разложения на множители в группах кос и изучаются с точностью до некоторых преобразований, называемых в настоящее время преобразованиями Гурвица. Мы предлагаем изучать их с помошью подходяших полугрупп над группами кос, так что гурвищ-эквивалентные разложения на множители соответствуют элементам этих полугрупп. В некоторых случаях (например, в случае топологических кривых Гурвица с несколькими особыми точками в слое; см. § 3 ) полезно переходить на второй уровень, т.е. рассматривать полугруппы разложений на множители над полугруппами первого уровня.

Работа первого автора была частично поддержана грантами INTAS-OPEN-00-0259, 000269 и РФФИ (грант № 02-01-00786). Второй автор является участником исследовательских групп EDGE и RAAG, поддержанных European Human Potential Program.

(C) Вик.С. Куликов, В.М. ХАРлАмов, 2003 
По мнению авторов, язык полугрупп упрощает построение и изучение объектов, заданных с помошью брэйд-монодромных разложений на множители. В качестве примера дано почти чисто алгебраическое доказательство недавнего результата С. Оревкова [19], которьй утверждает реализуемость любого брэйдмонодромного разложения на множители как bmf алгебраической кривой над диском (и который обобшает теорему Рудольфа [20] об алгебраической реализуемости квазиположительных кос). В нашей конструкции кривая расположена в некоторой линейчатой поверхности и имеет только простейшие точки ветвления вне рассматриваемого диска. В случае произвольных (не обязательно круговых) дисков эта конструкция позволяет ограничить степени коэффициентов многочлена, задающего кривую. С другой стороны, мы показываем, используя примеры Мойшезона [17], что для круговых дисков не существует таких оценок сверху, зависящих только от типов множителей, входящих в bmf.

Разложение на множители брэйд-монодромии проективной кривой, лежащей в линейчатой поверхности $\Sigma_{N}$, является разложением элемента $\Delta^{2 N}$, где $\Delta-$ так называемый элемент Гарсайда и $N$ - “степень линейчатости". Множители этого разложения соответствуют критическим точкам ограничения проекции на кривую. Как известно, в противоположность разложениям на множители брэйдмонодромии над диском, не любое проективное bmf может быть реализовано как bmf алгебраической кривой (см. [17]). С другой стороны, каждое проективное bmf может быть реализовано в классе кривых Гурвица. Мы доказываем, что тип брэйд-монодромного разложения на множители (под типом мы подразумеваем орбиту естественного действия групшы кос сопряжением, см. $\S 1$ ) различает с точностью до $H$-изотопии (т.е. изотопии в классе кривых Гурвица) кривые Гурвица с особыми точками вида $w^{k}=z^{n}$ по крайней мере в случае, когда критические значения проекции различных критичеких точек не совпадают друг с другом. Также мы показываем, что любая такая кривая Гурвица $H$-изотопна почти алгебраической кривой Гурвица, т.е. такой, которая может быть задана алгебраическим уравнением над некоторым диском, содержащим все критические значения проекции.

В $\S 4$ приведены несколько замечаний об изотопиях $J$-голоморфных кривых в $\mathbb{C P}^{2}$. В частности, используя известные результаты о $J$-голоморфных кривых и результаты, полученные в $\S 3$, мы показываем, что многие проблемы симплектической изотопии сводятся к чисто алгебраическим проблемам, связанным с изучением типов брэйд-монодромных разложений на множители, и доказываем, что две каспидальные $J$-голоморфные кривые симплектически изотопны тогда и только тогда, когда они имеют один и тот же тип брэйд-монодромного разложения на множители. Мы также даем bmf-критерий алгебраичности для нодальных симплектических поверхностей в $\mathbb{C P}^{2}$ : нодальная симплектическая поверхность симплектически изотопна алгебраической кривой тогда и только тогда, когда ee bmf является частичной регенерацией разложения на множители с множителями, сопряженными квадрату стандартной образующей группы кос.

Статья организована следуюшим образом. В $\S 1$ введены и рассмотрены полугрупшы разложений на множители: даны основные конструкции, исследованы их функториальные свойства и применены к групшам кос. Для полугрупп над группой кос введено понятие стабильного равенства и доказано, что два элемента с сопряженными множителями являются стабильно равными тогда и только тогда, когда они являются разложениями на множители одного и того же элемента в группе 
кос. Затем этот результат использован в $\S 2$ для доказательства упоминавшейся вьше обобщенной теоремы Рудольфа. В $\S 3$ введено понятие $H$-изотопии. Здесь, кроме кривых Гурвища и почти алгебраических кривых, имеющих алгебраические особенности, рассмотрены так называемые топологические кривые Гурвица, которые уже могут иметь произвольные конусообразные особенности. Кроме того, введено понятие конусообразных особенностей нерасщепляемого типа и доказано, что две топологические кривые одинаковой степени с неращепляемыми особыми точками, каждые две из которых лежат в разных слоях проекции, являются $H$-изотопными тогда и только тогда, когда эти кривые имеют один и тот же тип брэйд-монодромного разложения на множители. В $\S 4$ рассмотрен симплектический случай.

Авторы благодарны Е. Арталь-Бартоло, С. Немировскому и В. Шевчишину за полезные обсуждения и предложения. Настоящее исследование было начато в период пребывания первого автора в университете г. Страсбурга и окончено во время пребывания авторов в Математическом институте Обервольфаха в рамках программы RiP.

\section{§ 1. Исчисление разложений на множители}

1.1. Полугруппы разложений на множители. Набор $(S, B, \alpha, \lambda)$, где $S$ полугруппа, $B$ - группа и $\alpha: S \rightarrow B, \lambda: B \rightarrow \operatorname{Aut}(S)$ - гомоморфизмы, называется nолугруппой $S$ над $B$, если для всех $s_{1}, s_{2} \in S$ имеем

$$
s_{1} \cdot s_{2}=\lambda\left(\alpha\left(s_{1}\right)\right)\left(s_{2}\right) \cdot s_{1}=s_{2} \cdot \rho\left(\alpha\left(s_{2}\right)\right)\left(s_{1}\right)
$$

где $\rho(g)=\lambda\left(g^{-1}\right)$. Для пары полугрупп $\left(S_{1}, B_{1}, \alpha_{1}, \lambda_{1}\right)$ и $\left(S_{2}, B_{2}, \alpha_{2}, \lambda_{2}\right)$ соответственно над $B_{1}$ и $B_{2}$ пара гомоморфизмов $\left(h_{1}, h_{2}\right)$, где $h_{1}: S_{1} \rightarrow S_{2}$ и $h_{2}: B_{1} \rightarrow B_{2}$, называется гомоморфизмом полугрупп над группами, если:

(i) $h_{2} \circ \alpha_{S_{1}}=\alpha_{S_{2}} \circ h_{1}$;

(ii) $\lambda_{B_{2}}\left(h_{2}(g)\right)\left(h_{1}(s)\right)=h_{1}\left(\lambda_{B_{1}}(g)\right)(s)$ для всех $s \in S_{1}$ и всех $g \in B_{1}$.

Определенные ниже полугруппь разложений на множители являются основньми (для наших целей) примерами полугрупп над группами.

Пусть $\left\{g_{i}\right\}_{i \in I}$ - некоторое множество элементов группы $B$. Для каждого $i \in I$ обозначим через $O_{g_{i}} \subset B$ множество всех элементов из $B$, сопряженных элементу $g_{i}$ (т.е. орбиту элемента $g_{i}$ при действии группы $B$ внутренними автоморфизмами). Назовем объединение $X=\bigcup_{i \in I} O_{g_{i}} \subset B$ полным множеством әлементов, сопряженных әлементам $\left\{g_{i}\right\}_{i \in I}$, а пару $(B, X)$ - оснащенной әруппой.

Для любого полного множества $X$ сопряженных элементов имеем два естественных отображения $r=r_{X}: X \times X \rightarrow X$ и $l=l_{X}: X \times X \rightarrow X$, заданных $r(a, b)=b^{-1} a b$ и $l(a, b)=a b a^{-1}$ соответственно. Для каждой пары букв $a, b \in X$, $a \neq b$, обозначим через $R_{a, b ; r}$ и $R_{a, b ; l}$ следуюшие соотношения:

1) $R_{a, b ; r}$ имеет вид $a \cdot b=b \cdot r(a, b)$, если $b \neq \mathbf{1}$, и вид $a \cdot \mathbf{1}=a$ в противном случае;

2) $R_{a, b ; l}$ имеет вид $a \cdot b=l(a, b) \cdot a$, если $a \neq \mathbf{1}$, и вид $\mathbf{1} \cdot b=b$ в противном случае. Положив

$$
\mathscr{R}=\left\{R_{a, b ; r}, R_{a, b ; l} \mid(a, b) \in X \times X, a \neq b, \text { если } a \neq \mathbf{1} \text { или } b \neq \mathbf{1}\right\},
$$


определим с помощью множества соотношений $\mathscr{R}$ полугруппу

$$
S(B, X)=\langle x \in X: R \in \mathscr{R}\rangle \text {. }
$$

Определим также гомоморфизм $\alpha_{X}: S(B, X) \rightarrow B$, заданный $\alpha_{X}(x)=x$ для каждого $x \in X$. Поскольку $\alpha_{X}=\alpha_{B} \circ \mathrm{id}_{X, B}$, мы не будем делать различий между $\alpha_{X}$ и $\alpha_{B}$ и будем обозначать их через $\alpha$.

Кроме того, определим два действия $\lambda$ и $\rho$ группы $B$ на множестве $X$ :

$$
\begin{aligned}
& x \in X \mapsto \rho(g)(x)=g^{-1} x g \in X, \\
& x \in X \mapsto \lambda(g)(x)=g x g^{-1} \in X .
\end{aligned}
$$

Легко видеть, что множество соотношений $\mathscr{R}$ инвариантно относительно этих действий и, следовательно, $\rho$ и $\lambda$ определяют антигомоморфизм $\rho: B \rightarrow \operatorname{Aut}(S(B, X))$ (правое действие) и гомоморфизм $\lambda: B \rightarrow \operatorname{Aut}(S(B, X))$ (левое действие или действие сопряжением). Действие $\lambda(g)$ на $S(B, X)$ называется одновременным сопряжением на элемент $g$. Положим $\lambda_{S}=\lambda \circ \alpha$ и $\rho_{S}=\rho \circ \alpha$.

УТВЕРЖДЕНИЕ 1.1. Для каждого $g \in B$ и любых $x_{i}, x_{j} \in X$ имеем

(i) $\lambda(g)=\rho\left(g^{-1}\right)$;

(ii) $\alpha\left(r\left(x_{i}, x_{j}\right)\right)=x_{j}^{-1} x_{i} x_{j}$;

(iii) $\alpha\left(l\left(x_{i}, x_{j}\right)\right)=x_{i} x_{j} x_{i}^{-1}$;

(iv) $\rho_{S}\left(x_{i}\right)\left(x_{j}\right)=r\left(x_{j}, x_{i}\right)$;

(v) $\lambda_{S}\left(x_{i}\right)\left(x_{j}\right)=l\left(x_{i}, x_{j}\right)$;

(vi) $\rho\left(\alpha\left(x_{i}\right)^{-1}\right)\left(x_{j}\right)=l\left(x_{i}, x_{j}\right)$;

(vii) $\lambda\left(\alpha\left(x_{i}\right)^{-1}\right)\left(x_{j}\right)=r\left(x_{j}, x_{i}\right)$.

ДоКАЗАТЕЛЬСТВО следует из приведенных выше определений.

Из утверждения 1.1 следует, что $\left(S(B, X), B, \alpha_{X}, \lambda_{S}\right)$ является полугруппой над $B$. Назовем такие полугруппы полугруппами разложений на множители над $B$. Если $B$ фиксирована, то полугруппу $S(B, X)$ будем обозначать $S_{X}$. Через $x_{1} \cdot \ldots \cdot x_{n}$ обозначим элемент из $S_{X}$, определяемый словом $x_{1} \ldots x_{n}$.

Отметим, что $S:(B, X) \mapsto S(B, X)$ является функтором из категории оснащенных групп в категорию полугрупп. В частности, если $X \subset Y$ - два полных множества сопряженных в $B$ элементов, то тождественное отображение id: $B \rightarrow B$ определяет вложение $\operatorname{id}_{X, Y}: S(B, X) \rightarrow S(B, Y)$ полугрупп. Поэтому для каждой группы $B$ полугрупа $S_{B}=S(B, B)$ является универсальной полугруппой над $B$, т.е. каждая полугруппа $S_{X}$ над $B$ канонически вкладывается в $S_{B}$ с помошью $\operatorname{id}_{X, B}$.

Обозначим через $B_{X}$ подгруппу в $B$, порожденную образом гомоморфизма $\alpha$ : $S(B, X) \rightarrow B$, и для каждого $s \in S_{X}$ обозначим через $B_{s}$ подгруппу в $B$, порожденную образами $\alpha\left(x_{1}\right), \ldots, \alpha\left(x_{n}\right)$ множителей $x_{1}, \ldots, x_{n}$ разложения $s=$ $x_{1} \cdot \ldots \cdot x_{n}$.

УТВЕРЖДЕНИЕ 1.2. Подгруппа $B_{s}$ әруппь $B$ не зависит от представления элемента $s$ в виде слова из букв $x_{i}$ алфавита $X$.

ДокАЗАТЕльство следует из пп. (ii) и (iii) утверждения 1.1. 
ПрЕДЛОЖЕНИЕ 1.1. Для любого $X$ и любого $s \in S_{X}$ имеем:

(i) $\operatorname{ker} \lambda$ совпадает с иентрализатором $C_{X}$ группь $B_{X}$ в $B$;

(ii) если $\alpha(s)$ принадлежит чентру $C\left(B_{s}\right)$ группы $B_{s}$, то $\lambda(g)$ оставляет неподвижным әлемент $s \in S_{X}$, если $g \in B_{s}$.

ДокАЗАТЕЛЬСТво. Утверждение (i) очевидно.

Докажем утверждение (ii). Группа $B_{s}$ порождена в $B$ элементами $\alpha\left(x_{1}\right), \ldots$ $\ldots, \alpha\left(x_{n}\right)$, где $s=x_{1} \cdot \ldots \cdot x_{n}$ для некоторых $x_{i} \in X$. Поэтому для доказательства (ii) достаточно показать, что $\lambda_{S}\left(x_{i}\right)(s)=s$ для каждого $i=1, \ldots, n$ в случае, если $\alpha(s) \in C\left(B_{s}\right)$. Используя соотношения $x_{j} \cdot x_{i}=x_{i} \cdot r\left(x_{j}, x_{i}\right)$, можно сдвинуть $x_{i}$ влево и получить представление элемента $s$ в виде

$$
s=x_{i} \cdot \tilde{x}_{1} \cdot \ldots \cdot \tilde{x}_{n-1}=x_{i} \cdot \tilde{s} .
$$

Если $\alpha(s) \in C\left(B_{s}\right)$, то $\lambda_{S}(s)\left(x_{i}\right)=x_{i}$. Окончательно,

$$
\begin{aligned}
s & =l\left(\tilde{x}_{1}, x_{i}\right) \cdot \ldots \cdot l\left(\tilde{x}_{n-1}, x_{i}\right) \cdot x_{i}=\lambda_{S}\left(x_{i}\right)\left(\tilde{x}_{1} \cdot \ldots \cdot \tilde{x}_{n-1}\right) \cdot x_{i} \\
& =\lambda_{S}\left(x_{i}\right)(\tilde{s}) \cdot x_{i}=\lambda_{S}\left(\lambda_{S}\left(x_{i}\right)(\tilde{s})\right)\left(x_{i}\right) \cdot \lambda_{S}\left(x_{i}\right)(\tilde{s})=\lambda_{S}\left(x_{i} \cdot \tilde{s}\right)\left(x_{i}\right) \cdot \lambda_{S}\left(x_{i}\right)(\tilde{s}) \\
& =x_{i} \cdot \lambda_{S}\left(x_{i}\right)(\tilde{s})=\lambda_{S}\left(x_{i}\right)\left(x_{i}\right) \cdot \lambda_{S}\left(x_{i}\right)(\tilde{s})=\lambda_{S}\left(x_{i}\right)(s) .
\end{aligned}
$$

Рассмотрим два полных множества $X_{1}, X_{2}$ сопряженных элементов в $B$ и ассоциированные с ними полугруппы $S_{X_{1}}$ и $S_{X_{2}}$. Отображение $\psi: X_{2} \rightarrow S_{X_{1}}$ может быть доопределено до гомоморфизма $\psi: S_{X_{2}} \rightarrow S_{X_{1}}$ тогда и только тогда, когда для любых $x_{i}, x_{j} \in X_{2}$ равенства

$$
\begin{aligned}
& \psi\left(x_{i}\right) \cdot \psi\left(x_{j}\right)=\psi\left(x_{j}\right) \cdot \psi\left(r\left(x_{i}, x_{j}\right)\right) \\
& \psi\left(x_{i}\right) \cdot \psi\left(x_{j}\right)=\psi\left(l\left(x_{i}, x_{j}\right)\right) \cdot \psi\left(x_{i}\right)
\end{aligned}
$$

имеют место в $S_{X_{1}}$.

Скажем, что гомоморфизм $\psi$ определен над $B$, если $\alpha_{X_{2}}(x)=\alpha_{X_{1}}(\psi(x))$ для всех $x \in X_{2}$.

ПримеР 1.1. Пусть $X_{1}$ - полное множество элементов, сопряженных $x_{1} \in B$, и $X_{2}$ - полное множество элементов, сопряженных $x_{1}^{2}$. Предположим, что отображение $\phi: X_{1} \rightarrow X_{2}$, заданное формулой $\phi(x)=x^{2}$ для $x \in X_{1}$, является взаимно однозначньм. Тогда отображение $\psi: X_{2} \rightarrow S_{X_{1}}$, заданное формулой $\psi(x)=\phi^{-1}(x) \cdot \phi^{-1}(x)$, определяет гомоморфизм $\psi: S_{X_{2}} \rightarrow S_{X_{1}}$ над $B$.

ПримеР 1.2. Пример 1.1 может быть обобщен следующим образом. Выберем множество $\left\{x_{1}, \ldots, x_{n}\right\}$ элементов в полном множестве сопряженных элементов $X_{1} \subset B$ и множество произведений $s_{j}\left(x_{1}, \ldots, x_{n}\right)=x_{i_{1}(j)} \cdot \ldots \cdot x_{i_{m(j)}(j)} \in S_{X_{1}}$, $j=1, \ldots, k$. Рассмотрим $X_{2}=O_{\bar{s}_{1}} \cup \cdots \cup O_{\bar{s}_{k}}$, где $O_{\bar{s}_{j}}-$ полное множество элементов, сопряженных элементам $\bar{s}_{j} \alpha\left(s_{j}\right) \in B$. Предположим, что $\bar{s}_{i}$ и $\bar{s}_{j}$ не сопряжены в $B$ для $i \neq j$. Тогда отображение $X_{2} \rightarrow S_{X_{1}}$, заданное следуюшим образом:

$$
g \bar{s}_{j} g^{-1} \mapsto \lambda(g)\left(s_{j}\right) \in S_{X_{1}},
$$

может быть однозначно продолжено до гомоморфизма $r: S_{X_{2}} \rightarrow S_{X_{1}}$, определенного над $B$. Гомоморфизм $r$ называется регенерацией множества элементов $\left\{s_{j}\right\}$. 
В $\S 4$ будем использовать некоторое обобщение понятия регенерации (которое уже не будет гомоморфизмом), определяемое следуюшим образом. В обозначениях примера 1.2 положим $Z=X_{1} \cup X_{2}$ и рассмотрим элемент $z=z_{1} \cdot z_{2} \in S_{Z}$, где $z_{1} \in S_{X_{2}}, z_{2} \in S_{Z}$. Элемент

$$
\bar{z}=r\left(z_{1}\right) \cdot z_{2} \in S_{Z}
$$

назьвается частичной регенерацией элемента $z$.

Конструкция полугрупा $S(B, X)$ может быть итерирована. Именно, можно рассмотреть действие сопряжения группы $B$ на $S(B, X)$, выбрать некоторое множество $Y$, являющееся объединением орбит этого действия, и ввести полугруппу $S(S(B, X), Y)$ как полугруппу, порожденную буквами $s$ алфавита $Y$, связанными соотношениями

$$
\begin{aligned}
& s_{i}{ }^{\overline{ }} s_{j}=s_{j}{ }^{\overline{ }} \rho_{S}\left(s_{j}\right)\left(s_{i}\right), \\
& s_{i} \cdot s_{j}=\lambda_{S}\left(s_{i}\right)\left(s_{j}\right)^{\bar{*}} s_{i}
\end{aligned}
$$

для всех $s_{i}, s_{j} \in Y$.

Кроме того, можно определить гомоморфизм $\beta_{S}: S(S(B, X), Y) \rightarrow S(B, X)$, переводящий $s=s_{1}{ }^{\top} \ldots{ }^{\top} s_{n} \in S(S(B, X), Y)$ в $s_{1} \cdot \ldots \cdot s_{n} \in S(B, X)$, и действие сопряжением $\lambda: B \rightarrow \operatorname{Aut}(S(S(B, X), Y)$ ) (автоморфизм $\lambda(g)$ действует как одновременное сопряжение на элемент $g$ ), а также связанные с ними гомоморфизм $\beta=\alpha \circ \beta_{S}$ и действия $\lambda_{S}=\lambda \circ \alpha_{S}: S(B, X) \rightarrow \operatorname{Aut}(S(S(B, X), Y))$, $\lambda_{S, S}=\lambda \circ \beta: S(S(B, X), Y) \rightarrow \operatorname{Aut}(S(S(B, X), Y))$ соответственно полугрупп $S(B, X)$ и $S(S(B, X), Y)$ на $S(S(B, X), Y)$. Правые действия $\rho, \rho_{S}$ и $\rho_{S, S}$ соответственно группы $B$ и полугрупп $S(B, X)$ и $S(S(B, X), Y)$ на $S(S(B, X), Y)$ определяются аналогично.

Отметим, что если $X$ является подмножеством $Y$, то имеется естественное вложение полугруппы $S_{X}=S(B, X)$ в $S\left(S_{X}, Y\right)$. Более того, сушествует естественное вложение $S\left(S_{X}, Y\right)$ в универсальную полугруппу $S\left(S_{B}\right)=S\left(S_{B}, S_{B}\right)$ над $S_{B}$. Таким образом, полугруппа $S_{X}$ над $B$ может быть рассмотрена как подполугруппа полугруппы $S\left(S_{B}\right)$, и поэтому, обозначив снова операцию ‘ в $S\left(S_{B}\right)$ через ·, мы не придем к противоречию. Отметим, что $S\left(S_{X}, X\right)$ естественным образом изоморфна полугруппе $S_{X}$.

1.2. Эквивалентность Гурвица. Пусть, как и вьше, $Y$ - объединение орбит действия сопряжением $\lambda$ групшы $B$ на $S(B, X)$. Упорядоченное множество

$$
\left\{y_{1}, \ldots, y_{n} \mid y_{i} \in Y\right\}, \quad n \in \mathbb{Z}
$$

называется разложением на множители элемента $g=\beta\left(y_{1}\right) \ldots \beta\left(y_{n}\right) \in B$ в множестве $Y$. Обозначим $F_{X, Y}=\bigcup_{n} Y^{n}$ множество всех возможных разложений на множители элементов из $B$ в $Y$ для всех $n \in \mathbb{N}$. Существует естественное отображение $\varphi: F_{X, Y} \rightarrow S(S(B, X), Y)$, заданное формулой

$$
\varphi\left(\left\{y_{1}, \ldots, y_{n}\right\}\right)=y_{1} \cdot \ldots \cdot y_{n} .
$$

Преобразование, которое заменяет в $\left\{y_{1}, \ldots, y_{n}\right\}$ некоторые два соседних множителя $\left(y_{i}, y_{i+1}\right)$ на $\left(y_{i+1}, \rho_{S}\left(y_{i+1}\right)\left(y_{i}\right)\right)$ или $\left(\lambda_{S}\left(y_{i}\right)\left(y_{i+1}\right), y_{i}\right)$ и сохраняет все остальные множители, называется преобразованием Гурвица. Два разложения на множители называются гурвиц-эквивалентнымми, если одно из них может быть получено из другого с помощью конечного числа преобразований Гурвица. 
УТВЕРЖДЕНИЕ 1.3. Два разложения на множители $y=\left\{y_{1}, \ldots, y_{n}\right\}$ u $z=$ $\left\{z_{1}, \ldots, z_{n}\right\}$ гурвич-эквивалентны тогда и только тогда, когда $\varphi(y)=\varphi(z)$.

\section{ДОКАЗАТЕЛЬСТВО очевИдно.}

ЗАмечАниЕ 1.1. Далее согласно утверждению 1.3 будем отождествлять классыгурвищ-эквивалентных разложений на множители в $Y$ с их образами в $S\left(S_{X}, Y\right)$, а в случае $Y=X$ будем отождествлять полугруппу $S\left(S_{X}, Y\right)$ с $S_{X}$.

1.3. Полугруппы над группами кос и стабильная эквивалентность. В этом пункте группа $B=B_{m}$ является группой кос с $m$ нитями. Зафиксируем множество $\left\{a_{1}, \ldots, a_{m-1}\right\}$ так называемых стандартных образующих, т.е. образуюших, связанных соотношениями

$$
\begin{aligned}
a_{i} a_{i+1} a_{i} & =a_{i+1} a_{i} a_{i+1}, \quad 1 \leqslant i \leqslant n-1, \\
a_{i} a_{k} & =a_{k} a_{i}, \quad|i-k| \geqslant 2 .
\end{aligned}
$$

Обозначим через $B_{m}^{+}$полугруппу, порожденную теми же буквами, связанными теми же соотношениями.

ТЕОРЕМА ГАРСАЙДА [8]. Естественный гомоморфизм $i: B_{m}^{+} \rightarrow B_{m}$ является вложением.

Исходя из этой теоремы, отождествим полугруппу $B_{m}^{+}$с ее образом $i\left(B_{m}^{+}\right)$в $B_{m}$ и назовем образы $i(g)$ элементов $g \in B_{m}^{+}$положительными элементами группы $B_{m}$.

Обозначим через $A_{k}=A_{k}(m), k \geqslant 0$, полное множество элементов, сопряженных элементу $a_{1}^{k+1}$ в группе $B_{m}$ (напомним, что все образуюшие $a_{1}, \ldots, a_{m-1}$ сопряжены друг другу). Рассмотрим полугруппу $S_{A_{0}}$ как подполугруппу универсальной полугруппы $S_{B_{m}}$ над $B_{m}$. Положительное слово $g=a_{i_{1}} \ldots a_{i_{n}}$ в алфавите $\left\{a_{1}, \ldots, a_{n}\right\}$ определяет элемент $\bar{g}\left(a_{1}, \ldots, a_{m-1}\right)=a_{i_{1}} \cdot \ldots \cdot a_{i_{n}} \in S_{A_{0}}$. С другой стороны, $g$ определяет элемент $\tilde{g}=a_{i_{1}} \ldots a_{i_{n}}$ в $B_{m}^{+}$.

Лемма 1.1 [3]. Отображение $\nu: B_{m}^{+} \rightarrow S_{A_{0}}$, заданное формулой $\nu(\tilde{g})=\bar{g}$, является инбективным гомоморфизмом полугрупп.

ДокАЗАТЕЛЬСтво. Чтобы показать, что $\nu$ является гомоморфизмом, достаточно проверить, что соотношения (1.1) и (1.2) имеют место в $S_{A_{0}}$. Имеем

$$
\begin{aligned}
a_{i} \cdot a_{i+1} \cdot a_{i} & =a_{i+1} \cdot\left(a_{i+1}^{-1} a_{i} a_{i+1}\right) \cdot a_{i}=a_{i+1} \cdot a_{i} \cdot\left(a_{i}^{-1} a_{i+1}^{-1} a_{i} a_{i+1} a_{i}\right) \\
& =a_{i+1} \cdot a_{i} \cdot\left(a_{i}^{-1} a_{i+1}^{-1} a_{i+1} a_{i} a_{i+1}\right)=a_{i+1} \cdot a_{i} \cdot a_{i+1}
\end{aligned}
$$

для $1 \leqslant i \leqslant n-1$ и

$$
a_{i} \cdot a_{k}=a_{k} \cdot\left(a_{k}^{-1} a_{i} a_{k}\right)=a_{k} \cdot a_{i}
$$

для $|i-k| \geqslant 2$. Гомоморфизм $\nu$ инъективен, так как согласно теореме Гарсайда $\alpha_{B} \circ \nu$ является тождественным изоморфизмом.

Пусть $\Delta=\Delta_{m}$ - так называемый әлемент Гарсайда:

$$
\Delta=\left(a_{1} \ldots a_{m-1}\right) \ldots\left(a_{1} a_{2} a_{3}\right)\left(a_{1} a_{2}\right) a_{1} .
$$

Хорошо известно, что $\Delta^{2}=\left(a_{1} \ldots a_{m-1}\right)^{m}$ порождает центр групшы $B_{m}$. Обозначим через $\delta^{2}=\delta_{m}^{2}$ элемент в $S_{A_{0}} \subset S_{B_{m}}$, равный

$$
\delta^{2}=\left(a_{1} \cdot \ldots \cdot a_{m-1}\right)^{m} .
$$


ЛЕмма 1.2. Элемент $\delta^{2}$ неподвижен при действии сопряжением группь $B_{m}$ на $S_{B_{m}}$, m.е. $\rho(g)\left(\delta^{2}\right)=\delta^{2}$ для любого $g \in B_{m}$.

ДокАЗАТЕЛЬСтво следует из равенства $\alpha\left(\delta^{2}\right)=\Delta^{2}$ и предложения 1.1 ,(ii), примененного к $s=\delta^{2}$ (для которого $\left.\left(B_{m}\right)_{s}=B_{m}\right)$.

Исследуя топологические кривые Гурвища (см. п. 3.2), будем использовать некоторое расширение $\widetilde{S}_{B_{m}}$ полугрупшы $S_{B_{m}}$, которое определяется следующим образом. Каждому элементу $I$, принадлежашему множеству $\mathscr{I}$ всех подмножеств множества $\{1, \ldots, m\}$, сопоставим символ $\mathbf{1}_{I}$. Рассмотрим полугруппу $\widetilde{S}_{B_{m}}$, порожденную парами $\left(g, \mathbf{1}_{I}\right), g \in B_{m}$ и $I \in \mathscr{I}$, со следуюшими соотношениями между ними:

$$
\begin{aligned}
& \left(g_{1}, \mathbf{1}_{I_{1}}\right) \cdot\left(g_{2}, \mathbf{1}_{I_{2}}\right)=\left(g_{1} g_{2} g_{1}^{-1}, \mathbf{1}_{\sigma\left(g_{1}\right)\left(I_{2}\right)}\right) \cdot\left(g_{1}, \mathbf{1}_{I_{1}}\right) \\
& \left(g_{1}, \mathbf{1}_{I_{1}}\right) \cdot\left(g_{2}, \mathbf{1}_{I_{2}}\right)=\left(g_{2}, \mathbf{1}_{I_{2}}\right) \cdot\left(g_{2}^{-1} g_{1} g_{2}, \mathbf{1}_{\sigma\left(g_{2}^{-1}\right)\left(I_{1}\right)}\right)
\end{aligned}
$$

для всех $g_{1}, g_{2} \in B_{m}$ и всех $I_{1}, I_{2} \in \mathscr{I}$, где $\sigma$ - действие на $\mathscr{I}$, индуцированное естественным гомоморфизмом из $B_{m}$ в симметрическую группу $\Sigma_{m}$, действующую на $\{1, \ldots, m\}$. Чтобы продолжить действия $\lambda$ и $\rho$ группы $B_{m}$ на $S_{B_{m}}$ до действия на $\widetilde{S}_{B_{m}}$, положим

$$
\lambda(b)\left(\left(g, \mathbf{1}_{I}\right)\right)=\left(\lambda(b)(g), \mathbf{1}_{\sigma(b)(I)}\right)
$$

и $\rho(g)=\lambda\left(g^{-1}\right)$ для $b, g \in B_{m}$ и $I \in \mathscr{I}$. Продолжим гомоморфизм $\alpha$, положив

$$
\alpha\left(\left(g, \mathbf{1}_{I}\right)\right)=g \in B_{m}
$$

для всех $I$. Отметим, что отображение $g \mapsto\left(g, \mathbf{1}_{\varnothing}\right)$ продолжается до вложения полугруппы $S_{B_{m}}$ в полугруппу $\widetilde{S}_{B_{m}}$ над $B_{m}$.

Обозначим через $B_{k, i}, k+i \leqslant m$, подгруппу группы кос $B_{m}$, порожденную элементами $a_{i+1}, \ldots, a_{i+k-1}$, составляющими часть фиксированного множества стандартных образующих $a_{1}, \ldots, a_{m-1}$ группы $B_{m}$. Скажем, что индекс сплетения элемента $b \in B_{m}$ равен $l(b)=k$, если $k$ является наименьшим числом таким, что $b$ сопряжен в $B_{m}$ некоторому элементу из $B_{k, 0}$. Для пары $\left(B_{n, i}, b\right), b \in B_{n, i}$ и $l(b)=k$, элемент $\tilde{g}=\left(\bar{b}, \mathbf{1}_{\{i+k+1, \ldots, i+n\}}\right) \in \widetilde{S}_{B_{m}}$ называется стандартной tbmf-формой элемента $b \in B_{n, i}$, если $\bar{b} \in B_{k, i} \subset B_{n, i}$ сопряжен элементу $b$ в $B_{m}$ (если $i+k \geqslant n$, то $\{i+k+1, \ldots, i+n\}=\varnothing)$.

Теперь каждой конечной последовательности целых чисел $k_{1}, \ldots, k_{t}$ таких, что $k_{1}+\cdots+k_{t} \leqslant m, k_{1} \geqslant 2, \ldots, k_{t} \geqslant 2$, сопоставим последовательность подг рупп $B_{k_{i}, k_{1}+\cdots+k_{i-1}}, 1 \leqslant i \leqslant t$, групшы $B_{m}$. Рассмотрим подмножества $T_{k_{1}, \ldots, k_{t}}$ в $\widetilde{S}_{B_{m}}$, состоящие из произведений $s=\tilde{g}_{1} \cdot \ldots \cdot \tilde{g}_{t}$, где $\tilde{g}_{i} \in \widetilde{S}_{B_{m}}$ - стандартные tbmf-формы элементов из $B_{k_{i}, k_{1}+\cdots+k_{i-1}}, \quad 1 \leqslant i \leqslant t$. Определим $T=T_{m}$ как объединение $\bigcup \lambda(g) T_{k_{1}, \ldots, k_{t}}$ по всем $g \in B_{m}$ и всем последовательностям цельх чисел $k_{1}, \ldots, k_{t}$ таким, что $k_{1}+\cdots+k_{t} \leqslant m$. Отметим, что для любой подстановки $\sigma \in \Sigma_{t}$ и любого $\tilde{g}_{1} \cdot \ldots \cdot \tilde{g}_{t} \in T_{k_{i}, k_{1}+\cdots+k_{i-1}}$ имеет место равенство

$$
\tilde{g}_{1} \cdot \ldots \cdot \tilde{g}_{t}=\tilde{g}_{\sigma(1)} \cdot \ldots \cdot \tilde{g}_{\sigma(t)}
$$


Элементы из $T$ называются tbm-разложениями на множители, полугруппа $\mathscr{T}=\mathscr{T}_{m}=S\left(\widetilde{S}_{B_{m}}, T\right)$ - полугруппой tbm-разложений на множсители. Скажем, что два tbm-разложения на множители имеют один и тот же тип разложения на множители, если они принадлежат одной и той же орбите действия сопряжением группы $B_{m}$.

Группа $B_{m}$ как множество может быть представлена в виде несвязного объединения (по всем $k, 1 \leqslant k \leqslant m$ ) орбит множеств $T_{k, \varnothing}=\left\{\left(b, \mathbf{1}_{\varnothing}\right) \mid l(b)=k\right\} \subset T_{k}$ при действии сопряжением группы $B_{m}$. Это представление определяет вложение $i: S_{B_{m}} \rightarrow \mathscr{T}$ полугрупп над $B_{m}$. Таким образом, когда это не приводит к двусмысленности, будем отождествлять $S_{B_{m}}$ с ее образом $i\left(S_{B_{m}}\right) \subset \mathscr{T}$.

Скажем, что элемент $s_{1} \in \mathscr{T}$ (в частности, $s_{1} \in S_{B_{m}}$ ) стабильно равен элементу $s_{2} \in \mathscr{T}$ (соответственно, $s_{2} \in S_{B_{m}}$ ), если найдется такое целое число $n \geqslant 1$, что $s_{1} \cdot\left(\delta^{2}\right)^{n}=s_{2} \cdot\left(\delta^{2}\right)^{n}$ в $\mathscr{T}$.

Теорема 1.1. Пусть $O_{x_{1}}, \ldots, O_{x_{n}}$ - орбиты әлементов $x_{1}, \ldots, x_{n} \in T$ при действии сопряжением группы $B_{m}$ на $T$. Для любых $y_{i} \in O_{x_{i}}, \quad 1 \leqslant i \leqslant n, u$ для любой перестановки $\sigma \in \Sigma_{n}$ әлементы $s_{1}=x_{1} \ldots \cdot x_{n} u s_{2}=y_{\sigma(1)} \cdot \ldots \cdot y_{\sigma(n)}$ стабильно равны в $\mathscr{T}$ тогда и только тогда, когда $\beta\left(s_{1}\right)=\beta\left(s_{2}\right)$.

ЗАмЕчАниЕ 1.2. Утверждение теоремы 1.1 остается справедливым, если заменить $T$ на любое полное множество $X \subset S_{B_{m}}$, содержащее $A_{0}$, а $\mathscr{T}$ заменить на $S\left(S_{B_{m}}, X\right)$.

ДОКАЗАТЕЛЬСТВО ТЕОРЕМЫ 1.1. Очевидно, что если $s_{1}$ и $s_{2}$ стабильно равны в $\mathscr{T}$, то $\beta\left(s_{1}\right)=\beta\left(s_{2}\right)$.

Пусть $\beta\left(s_{1}\right)=\beta\left(s_{2}\right)$. Поскольку любая подстановка является произведением транспозиций, можно предположить, что $\sigma=\mathrm{id}$. Действительно, для любых $g_{1}, g_{2} \in \mathscr{T}$ имеем соотношение $g_{1} \cdot g_{2}=g_{2} \cdot \rho\left(g_{2}\right)\left(g_{1}\right)$ в $\mathscr{T}$, в котором $g_{1}$ и $\rho\left(g_{2}\right)\left(g_{1}\right)$ принадлежат одной и той же орбите. Поэтому из этих соотношений вытекает, что найдется разложение $s_{2}=\tilde{y}_{1} \cdot \ldots \cdot \tilde{y}_{n}$ с множителями $\tilde{y}_{i} \in O_{x_{i}}$.

Лемма 1.3 [8]. Для любого $g \in B_{m}$ найдутся положительные элементы $r_{1}, r_{2} \in B_{m}$ и челье числа $k, p \in \mathbb{Z}, \quad p \geqslant 1$, такие, что:

(i) $g=\Delta^{2 k} r_{1}$;

(ii) $g r_{2}=\Delta^{2 p}$.

ДокАЗАТЕЛьСтво следует из [8, теорема 5].

По лемме $1.3,(\mathrm{i})$, поскольку $x_{i}$ и $\tilde{y}_{i}$ принадлежат одной и той же орбите $O_{x_{i}}$, а $\Delta^{2}$ принадлежит центру группы $B_{m}$, можно найти положительные элементы $g_{i}$ такие, что $\tilde{y}_{i}=\rho\left(g_{i}^{-1}\right)\left(x_{i}\right)$. Применяя лемму 1.3 , (ii) к каждому $g_{i}$, можно найти положительные элементы $r_{i}$ и положительные целые числа $p_{i}$ такие, что $g_{i} r_{i}=$ $\Delta^{2 p_{i}}$. Согласно теореме Гарсайда, утверждению 1.1 и лемме 1.1 имеем $\bar{g}_{i} \cdot \bar{r}_{i}\left(\delta^{2}\right)^{p_{i}}$ в $S_{A_{0}} \subset S_{B_{m}} \subset \mathscr{T}$. Положим $p=p_{1}+\cdots+p_{n}$. Из предложения 1.1 ,(ii) следует, что для каждого $x \in S_{B_{m}}$ имеем равенство $x \cdot \delta^{2}=\delta^{2} \cdot x$ в $S_{B_{m}}$. Кроме того, $\rho\left(g_{i}^{-1}\right)\left(x_{i}\right) \cdot \bar{g}_{i}=\bar{g}_{i} \cdot x_{i}$. Следовательно,

$$
\begin{aligned}
s_{2} \cdot\left(\delta^{2}\right)^{p} & =\rho\left(g_{1}^{-1}\right)\left(x_{1}\right) \cdot \ldots \cdot \rho\left(g_{n}^{-1}\right)\left(x_{n}\right) \cdot\left(\delta^{2}\right)^{p} \\
& =\rho\left(g_{1}^{-1}\right)\left(x_{1}\right) \cdot\left(\delta^{2}\right)^{p_{1}} \cdot \ldots \cdot \rho\left(g_{n}^{-1}\right)\left(x_{n}\right) \cdot\left(\delta^{2}\right)^{p_{n}} \\
& =\rho\left(g_{1}^{-1}\right)\left(x_{1}\right) \cdot \bar{g}_{1} \cdot \bar{r}_{1} \cdot \ldots \cdot \rho\left(g_{n}^{-1}\right)\left(x_{n}\right) \cdot \bar{g}_{n} \cdot \bar{r}_{n} \\
& =\bar{g}_{1} \cdot x_{1} \cdot \bar{r}_{1} \cdot \ldots \cdot \bar{g}_{n} \cdot x_{n} \cdot \bar{r}_{n} .
\end{aligned}
$$


Рассмотрим сначала случай, когда все $x_{i}$ являются стандартными образующими. В этом случае, применяя теорему Гарсайда к $\alpha\left(s_{1}\right)\left(\Delta^{2}\right)^{p}=\alpha\left(s_{2}\right)\left(\Delta^{2}\right)^{p}$, получаем

$$
s_{1} \cdot\left(\delta^{2}\right)^{p}=\bar{g}_{1} \cdot x_{1} \cdot \bar{r}_{1} \cdot \ldots \cdot \bar{g}_{n} \cdot x_{n} \cdot \bar{r}_{n}
$$

в $\nu\left(B_{m}^{+}\right)$, что приводит к равенству $s_{1} \cdot\left(\delta^{2}\right)^{p}=s_{2} \cdot\left(\delta^{2}\right)^{p}$ в $\nu\left(B_{m}^{+}\right)$.

В общем случае все $\bar{g}_{i}$ и $\bar{r}_{i}$ принадлежат $\nu\left(B_{m}^{+}\right)$. Применяя соотношения $a_{i} \cdot x_{j}=$ $x_{j} \cdot \rho_{S, S}\left(x_{j}\right)\left(a_{i}\right)$, можно сдвинуть влево все $x_{i}$ и получить, что $s_{2} \cdot\left(\delta^{2}\right)^{p}=s_{1} \cdot s_{3}$, где $s_{3}=\prod\left(t_{i}^{-1} z_{i} t_{i}\right)$ и каждое $z_{i}$ является буквой в алфавите $\left\{a_{1}, \ldots, a_{m-1}\right\}$. Таким образом, существует положительное целое число $q$ такое, что $s_{3} \cdot\left(\delta^{2}\right)^{q}=\left(\prod z_{i}\right) \times$ $\left(\delta^{2}\right)^{q}$, и поэтому

$s_{2} \cdot\left(\delta^{2}\right)^{p+q}=s_{2} \cdot\left(\delta^{2}\right)^{p} \cdot\left(\delta^{2}\right)^{q}=s_{1} \cdot s_{3} \cdot\left(\delta^{2}\right)^{q}=s_{1} \cdot\left(\prod z_{i}\right) \cdot\left(\delta^{2}\right)^{q} \cdot s_{1} \cdot\left(\delta^{2}\right)^{p+q}$,

так как $\left(\delta^{2}\right)^{p+q}$ и $\left(\prod z_{i}\right) \cdot\left(\delta^{2}\right)^{q}$ принадлежат $\nu\left(B_{m}^{+}\right)$и $\alpha\left(\left(\prod z_{i}\right) \cdot\left(\delta^{2}\right)^{q}\right)=\left(\Delta^{2}\right)^{p+q}$.

Следуюшие две проблемы, по мнению авторов, являются открытыми.

ПРОБЛЕМА ГАРСАЙДА. Является ли гомоморфизм $\alpha: S_{A_{0}(m)} \rightarrow B_{m}$ вложением для каждого $m$ ? В частности, имеет ли уравнение $\alpha(s)=\Delta_{m}^{2}$ только одно решение: $s=\delta_{m}^{2}$ ?

ПроБлЕмА СЛов. Имеет ли проблема слов для $\mathscr{T}_{m}$ (соответственно, для $S_{B_{m}}$, для $\left.S_{A_{\leqslant 2}}, A_{\leqslant 2}=\bigcup_{k \leqslant 2} A_{k}(m)\right)$ положительное решение?

1.4. “Чисто нодальная" полугруппа. В этом пункте мы имеем дело с полугруппой $S_{A_{1}(m)}$. Зафиксируем стандартные образуюшие $\left\{a_{1}, \ldots, a_{m-1}\right\}$ группы $B_{m}$ и рассмотрим $B_{m-1}$ как подгруппу группы $B_{m}$, порожденную элементами $\left\{a_{1}, \ldots, a_{m-2}\right\}$. Положим

$$
\tilde{\delta}_{m}^{2}=\prod_{l=m}^{2} \prod_{k=1}^{l-1} z_{k, l}^{2} \in S_{A_{1}},
$$

где $z_{k, l}=\left(a_{l-1} \ldots a_{k+1}\right) a_{k}\left(a_{l-1} \ldots a_{k+1}\right)^{-1}$ для $k<l$ (здесь $\prod_{a}^{b}$ означает произведение слева направо, начиная с индекса $a$ и кончая индексом $b$ ). Как известно (см., например, [18]), $\alpha\left(\tilde{\delta}_{m}^{2}\right)=\Delta_{m}^{2}$ и

$$
\tilde{\delta}_{m}^{2}=\prod_{k=1}^{m-1} z_{k, m}^{2} \cdot \tilde{\delta}_{m-1}^{2} .
$$

Лемма 1.4. Имеем:

(i) $a_{k} z_{j, k}^{2} a_{k}^{-1}=z_{j, k+1}^{2}$;

(ii) $a_{k} z_{k, m}^{2} a_{k}^{-1}=z_{k+1, m}^{2}$;

(iii) $a_{k} z_{j, r}^{2} a_{k}^{-1}=z_{j, r}^{2}$, если либо $r<k$, либо $j<k$, либо $j>k+1$;

(iv) $a_{k} z_{k+1, m}^{2} a_{k}^{-1}=z_{k+1, m}^{-2} z_{k, m}^{2} z_{k+1, m}^{2}$.

ДоКАЗАТЕЛЬСТВО следует из соотношений (1.1) и (1.2) и определения элементов $z_{k, j}^{2}$. 
ПРЕДЛОЖЕНИЕ 1.2. Элемент $\tilde{\delta}_{m}^{2} \in S_{A_{1}}$ является неподвижным при действии группь $B_{m}$ сопряжением.

ДокАЗАТЕльСтво. Достаточно показать, что $\lambda\left(a_{i}\right)\left(\tilde{\delta}_{m}^{2}\right)=\tilde{\delta}_{m}^{2}$ для $i=1, \ldots$ $\ldots, m-1$. Докажем эти равенства индукцией по $m$.

По предположению индукции и по лемме 1.4 для $i<m-1$ имеем

$$
\begin{aligned}
\lambda\left(a_{i}\right)\left(\tilde{\delta}_{m}^{2}\right)= & \lambda\left(a_{i}\right)\left(\prod_{k=1}^{m-1} z_{k, m}^{2} \cdot \tilde{\delta}_{m-1}^{2}\right)= \\
= & \prod_{k=1}^{i-1} \lambda\left(a_{i}\right)\left(z_{k, m}^{2}\right) \cdot \lambda\left(a_{i}\right)\left(z_{i, m}^{2}\right) \cdot \lambda\left(a_{i}\right)\left(z_{i+1, m}^{2}\right) \\
& \cdot \prod_{k=i+2}^{m-1} \lambda\left(a_{i}\right)\left(z_{k, m}^{2}\right) \cdot \lambda\left(a_{i}\right)\left(\tilde{\delta}_{m-1}^{2}\right) \\
= & \prod_{k=1}^{i-1} z_{k, m}^{2} \cdot z_{i+1, m}^{2} \cdot\left(z_{i+1, m}^{-2} z_{i, m}^{2} z_{i+1, m}^{2}\right) \cdot \prod_{k=i+2}^{m-1} z_{k, m}^{2} \cdot \tilde{\delta}_{m-1}^{2}=\tilde{\delta}_{m}^{2} .
\end{aligned}
$$

Применяя снова лемму 1.4, получаем, что для $i=m-1$

$$
\begin{aligned}
\lambda\left(a_{m-1}\right)\left(\tilde{\delta}_{m}^{2}\right)= & \lambda\left(a_{m-1}\right)\left(\prod_{k=1}^{m-1} z_{k, m}^{2} \cdot \prod_{k=1}^{m-2} z_{k, m-1}^{2} \cdot \tilde{\delta}_{m-2}^{2}\right) \\
= & \prod_{k=1}^{m-2} \lambda\left(a_{m-1}\right)\left(z_{k, m}^{2}\right) \cdot \lambda\left(a_{m-1}\right)\left(z_{m-1, m}^{2}\right) \\
& \cdot \prod_{k=1}^{m-2} \lambda\left(a_{m-1}\right)\left(z_{k, m-1}^{2}\right) \cdot \lambda\left(a_{m-1}\right)\left(\tilde{\delta}_{m-2}^{2}\right) \\
= & \prod_{k=1}^{m-2}\left(a_{m-1} z_{k, m}^{2} a_{m-1}^{-1}\right) \cdot z_{m-1, m}^{2} \cdot \prod_{k=1}^{m-2}\left(a_{m-1} z_{k, m-1}^{2} a_{m-1}^{-1}\right) \cdot \tilde{\delta}_{m-2}^{2} \\
= & z_{m-1, m}^{2} \cdot \prod_{k=1}^{m-2}\left(a_{m-1}^{-1} z_{k, m}^{2} a_{m-1}\right) \cdot \prod_{k=1}^{m-2} z_{k, m}^{2} \cdot \tilde{\delta}_{m-2}^{2} \\
= & z_{m-1, m}^{2} \cdot \prod_{k=1}^{m-2} z_{k, m-1}^{2} \cdot \prod_{k=1}^{m-2} z_{k, m}^{2} \cdot \tilde{\delta}_{m-2}^{2} \cdot
\end{aligned}
$$

Для завершения доказательства достаточно показать, что

$$
\prod_{k=1}^{m-2} z_{k, m}^{2} \cdot z_{m-1, m}^{2} \cdot \prod_{k=1}^{m-2} z_{k, m-1}^{2}=z_{m-1, m}^{2} \cdot \prod_{k=1}^{m-2} z_{k, m-1}^{2} \cdot \prod_{k=1}^{m-2} z_{k, m}^{2}
$$

Имеем

$$
t_{m}=\alpha\left(z_{m-1, m}^{2} \cdot \prod_{k=1}^{m-2} z_{k, m-1}^{2}\right)=a_{m-1}^{2} a_{m-2} \ldots a_{2} a_{1}^{2} a_{2} \ldots a_{m-2}
$$


и чтобы доказать равенство (1.3), достаточно показать, что

$$
t_{m} z_{k, m}^{2} t_{m}^{-1}=z_{k, m}^{2}
$$

для $k=1, \ldots, m-2$. Индукцией по $m$, применяя соотношения (1.1) и (1.2), имеем для $k \leqslant m-3$

$$
\begin{aligned}
t_{m} z_{k, m}^{2} t_{m}^{-1}= & \left(a_{m-1}^{2} a_{m-2}^{-1} t_{m-1} a_{m-2}\right) z_{k, m}^{2}\left(a_{m-1}^{2} a_{m-2}^{-1} t_{m-1} a_{m-2}\right)^{-1} \\
= & \left(a_{m-1}^{2} a_{m-2}^{-1} t_{m-1} a_{m-2} a_{m-1} a_{m-2}\right) \\
& \times z_{k, m-2}^{2}\left(a_{m-1}^{2} a_{m-2}^{-1} t_{m-1} a_{m-2} a_{m-1} a_{m-2}\right)^{-1} \\
= & \left(a_{m-1}^{2} a_{m-2}^{-1} t_{m-1} a_{m-1} a_{m-2} a_{m-1}\right) \\
& \times z_{k, m-2}^{2}\left(a_{m-1}^{2} a_{m-2}^{-1} t_{m-1} a_{m-1} a_{m-2} a_{m-1}\right)^{-1} \\
= & \left(a_{m-1}^{2} a_{m-2}^{-1} t_{m-1} a_{m-1} a_{m-2}\right) z_{k, m-2}^{2}\left(a_{m-1}^{2} a_{m-2}^{-1} t_{m-1} a_{m-1} a_{m-2}\right)^{-1} \\
= & \left(a_{m-1}^{2} a_{m-2}^{-1} t_{m-1} a_{m-1}\right) z_{k, m-1}^{2}\left(a_{m-1}^{2} a_{m-2}^{-1} t_{m-1} a_{m-1}\right)^{-1} \\
= & \left(a_{m-1}^{2} a_{m-2} a_{m-1} a_{m-2}^{-2} t_{m-1}\right) z_{k, m-1}^{2}\left(a_{m-1}^{2} a_{m-2} a_{m-1} a_{m-2}^{-2} t_{m-1}\right)^{-1} \\
= & \left(a_{m-1} a_{m-1} a_{m-2} a_{m-1} a_{m-2}^{-2}\right) \\
& \times z_{k, m-1}^{2}\left(a_{m-1} a_{m-1} a_{m-2} a_{m-1} a_{m-2}^{-2}\right)^{-1} \\
= & \left(a_{m-1} a_{m-1} a_{m-2} a_{m-1} a_{m-2}^{-1}\right) \\
& \times z_{k, m-1}^{2}\left(a_{m-1} a_{m-1} a_{m-2} a_{m-1} a_{m-2}^{-1}\right)^{-1} \\
= & \left(a_{m-2} a_{m-1}\right) z_{k, m-1}^{2}\left(a_{m-2} a_{m-1}\right)^{-1} \\
= & \left(a_{m-2} a_{m-1} a_{m-2}\right) z_{k, m-2}^{2}\left(a_{m-2} a_{m-1} a_{m-2}\right)^{-1} \\
= & \left(a_{m-1} a_{m-2} a_{m-1}\right) z_{k, m-2}^{2}\left(a_{m-1} a_{m-2} a_{m-1}\right)^{-1} \\
= & a_{m-1} z_{k, m-1}^{2} a_{m-1}^{-1}=z_{k, m}^{2} .
\end{aligned}
$$

Как и выше, проделав аналогичные вычисления, можно показать, что $t_{m} z_{m-2, m}^{2} t_{m}^{-1}=z_{m-2, m}^{2}$.

Следуюшая теорема является следствием из предложения 1.2 , а также следствия 4.1, теоремы 3.1 и замечания 4.1, которые будут доказаны ниже.

ТЕОРема 1.2. Пусть $A_{1} \subset B_{m}$ - полное множество әлементов, сопряженных элементу $a_{1}^{2}$. Тогда $\tilde{\delta}_{m}^{2}$ является единственным әлементом среди элементов $s \in S_{A_{1}}$ таких, что $\alpha(s)=\Delta_{m}^{2}$.

\section{§ 2. Существование многочлена с заданным над диском разложением монодромии на множители}

2.1. Локальная брэйд-монодромия над точкой (случай одного ростка). Здесь и далее мы обозначаем через $(z, w)$ стандартные координаты в $\mathbb{C}^{2}$. Доказательства (хорошо известных) утверждений, используемых здесь и далее, могут быть найдены, например, в [4].

Если росток $(C, o) \subset\left(\mathbb{C}^{2}, o\right)$, где $o=(0,0)$, приведенной комплексно-аналитической кривой не содержит росток кривой $z=0$, то он может быть задан уравнением

$$
P(z, w)=0
$$


где

$$
P(z, w)=w^{k}+\sum_{i=1}^{k} q_{i}(z) w^{k-i}
$$

и $q_{i}(z)$ - сходящиеся степенные ряды (т.е. $\left.q_{i}(z) \in \mathbb{C}\{z\}\right), q_{i}(0)=0$, и многочлен $w^{k}+\sum q_{i}(z) w^{k-i} \in \mathbb{C}\{z\}[w]$ не имеет кратных множителей. Поэтому можно выбрать мальй полидиск $D=D_{1} \times D_{2} \subset \mathbb{C}^{2}, D_{1}=D_{1}\left(\varepsilon_{1}\right)=\left\{z \in \mathbb{C}|| z \mid<\varepsilon_{1}\right\}$ и $D_{2}=D_{2}\left(\varepsilon_{2}\right)=\left\{w \in \mathbb{C}|| w \mid<\varepsilon_{2}\right\}$, такой, что $C$ является аналитическим множеством в каждой точке замыкания $\mathrm{Cl} D$ полидиска $D$, проекция на $z$-множитель $\mathrm{pr}=\mathrm{pr}_{1}: C \cap D \rightarrow D_{1}$ является собственным конечным отображением степени $k$ и $(z, w)=o$ является единственной критической точкой проекции $\mathrm{pr} \mid C \cap \mathrm{Cl} D$. Обратно, если $D$ - полидиск и $C-W$-подготовленньй росток в $D$, т.е. если $C$ - приведенная комплексно-аналитическая кривая с перечисленными выше свойствами относительно pr, то она может быть задана в $\mathrm{Cl} D$ уравнением такого типа. По определению $W$-подготовленный росток $(C, o)$ является алгебраическим (в координатах $(z, w))$ тогда и только тогда, когда $q_{i} \in \mathbb{C}[z]$ для каждого $i=1, \ldots, k$.

Чтобы определить брэйд-монодромию (монодромию в косах), выберем точку $u \in \partial D_{1}$ и положим $D_{2, u}=\operatorname{pr}^{-1}(u), K=K(u)=\left\{w_{1}, \ldots, w_{k}\right\}=D_{2, u} \cap$ $C$. Петля $\partial D_{1}$ с ориентацией против часовой стрелки и началом в $u$ поднимается в $\partial D \cap C$ как траектория движения $k$ различных точек $\operatorname{pr}_{2}\left(\left\{w_{1}(t), \ldots, w_{k}(t)\right\}\right)$ в $D_{2}$, начинающаяся и оканчивающаяся в $K$. Эта траектория определяет косу $b_{(C, o)} \in B_{k}=B_{k}\left[D_{2}, K\right]$, которая называется брэйд-монодромией ростка $(C, o)$ относительно проекиии pr. Отметим, что $l\left(b_{(C, o)}\right)=k$.

Линк ростка $(C, o)$ является положительным итерированным торическим зацеплением. Этот линк определяется парами Пюизо неприводимых компонент ростка $(C, o)$ и взаимными индексами пересечений компонент. Поэтому, как только мы выбираем в $B_{k}$ в качестве стандартных образующих положительные полуповороты $a_{1}, \ldots, a_{k-1}$, коса $b_{(C, o)}$ становится элементом, принадлежашим $B_{k}^{+}$(становится положительной косой). Назовем ее образ $\nu\left(b_{(C, o)}\right) \in B_{k}$ стандартной формой брәйд-монодромии ростка $(C, o)$ относительно pr. Стандартные образующие и, таким образом, стандартная форма $b_{(C, o)}$ определены однозначно $c$ точностью до сопряжения.

Топологический тип тройки $(D, C, \mathrm{pr})$ определяется ее стандартной формой брэйд-монодромии, верно и обратное утверждение. Кроме того, для каждой тройки $(D, C, \mathrm{pr})$ найдется константа $M=M_{(C, o)} \in \mathbb{N}$ такая, что топологический тип тройки $(D, C, \mathrm{pr})$ совпадает с топологическим типом особенности, заданной уравнением

$$
\bar{P}(z, w)=w^{k}+\sum \bar{q}_{i}(z) w^{k-i}=0,
$$

где $\bar{q}_{i}(z)=q_{i}(z)+z^{M} r_{i}(z)$ и $r_{i}(z)-$ произвольные аналитические функции. В частности, существует алгебраическая тройка, имеющая тот же топологический тип.

Топологический тип тройки $(D, C, \mathrm{pr})$ и, таким образом, стандартная форма ее брэйд-монодромии определяются ее разрешением особенности относительно $\mathrm{pr}$, т.е. композицией $\sigma$ таких раздутий $\sigma_{1}: U_{1} \rightarrow D, \ldots, \sigma_{n}: U_{n} \rightarrow U_{n-1}$ с центрами в точках, что $\sigma^{-1}(C \cup F)$ является дивизором с нормальными пересечениями, где 
$F=\{z=0\}$. Положим

$$
\begin{aligned}
& \sigma^{*}(C)=C^{\prime}+\sum_{i=1}^{n} c_{i} E_{i}, \\
& \sigma^{*}(F)=E_{0}+\sum_{i=1}^{n} a_{i} E_{i},
\end{aligned}
$$

где $C^{\prime}, E_{0}$ и $E_{i}, 1 \leqslant i \leqslant n$, являются собственными прообразами в $U_{n}$ ростка $C$, слоя $F$ и исключительных дивизоров раздутий $\sigma_{i}, 1 \leqslant i \leqslant n$, соответственно. В этих обозначениях в качестве упомянутой выше константы $M$ можно взять любое $m$ такое, что

$$
m\left(\sum a_{i} E_{i}\right)-\left(\sum c_{i} E_{i}\right)
$$

является строго положительным дивизором, т.е. для всех $i$ выполнены неравенства $m a_{i}-c_{i}>0$. Действительно, пусть $s$ - особая точка кривой $\sigma^{-1}(C \cup F)_{\mathrm{red}}$, принадлежашая $C^{\prime} \cap E_{i}$ для некоторого $i$. Выберем локальные координаты $\left(z_{i}, w_{i}\right)$ в окрестности точки $s$ так, что $z_{i}=0$ является уравнением $E_{i}$ и $w_{i}=0$-уравнение кривой $C^{\prime}$. Имеем

$$
\sigma^{*}(z)=z_{i}^{a_{i}}, \quad \sigma^{*}(P(z, w))=z_{i}^{c_{i}} w_{i}
$$

с точностью до функции, не обращающейся в нуль в точке $s$, и

$$
\sigma^{*}(\bar{P}(z, w))=\left(w_{i}+z_{i}^{M a_{i}-c_{i}} \sum \sigma^{*}\left(w^{k-j} r_{j}(z)\right)\right) z_{i}^{c_{i}} .
$$

Следовательно, росток, заданньй уравнением $\bar{P}(z, w)=0$, имеет то же самое разрешение особенностей, что и $(C, o)$.

2.2. Локальная брэйд-монодромия над точкой (случай нескольких ростков). Пусть теперь $C \subset \mathbb{C}^{2}-$ аффинная приведенная алгебраическая кривая, заданная в координатах $(z, w)$ уравнением

$$
w^{m}+\sum_{i=1}^{m} q_{i}(z) w^{m-i}=0, \quad q_{i} \in \mathbb{C}[z]
$$

Заметим, что любая аффинная алгебраическая кривая может быть задана такого типа уравнением после подходящей линейной замены координат.

Рассмотрим пересечение $C_{\varepsilon_{1}}=C \cap\left(D_{1}\left(\varepsilon_{1}\right) \times D_{2}\left(\varepsilon_{2}\right)\right), \varepsilon_{2}=\varepsilon_{1}^{-1}$. При условии $0<\varepsilon_{1} \ll 1$ проекция $\operatorname{pr}_{\mid \varepsilon_{\varepsilon_{1}}}: C_{\varepsilon_{1}} \rightarrow D_{1}=D_{1}\left(\varepsilon_{1}\right)$ является собственным отображением степени $m$ с единственным критическим значением $z=0$ (в противоположность ситуации, рассмотренной в п. 2.1 , здесь число критических точек может быть больше 1). Допуская общепринятую вольность изложения, назовем $C_{\varepsilon_{1}}$ ростком кривой $C$ над точкой 0 относительно pr. Как и в локальном случае (см. п. 2.1), зафиксируем точку $u \in \partial D_{1}$ и положим $D_{2, u}=\mathrm{pr}^{-1}(u)$, $K=K(u)=\left\{w_{1}, \ldots, w_{m}\right\}=D_{2, u} \cap C$. Снабжая $\partial D_{1}$ ориентацией против часовой стрелки, получим над $\partial D_{1}$, с помошью проекции $\operatorname{pr}_{2}\left(C \sim{ }_{\tilde{b}} \cap D_{1}\right)$, ориентированный след от движения $m$ точек в $D_{2}$ и, следовательно, косу $\tilde{b}_{\left(\varepsilon_{\varepsilon_{1}}, 0\right)} \in B_{m}=B_{m}\left[D_{2}, K\right]$. 
Пусть $\operatorname{pr}_{\mid C}^{-1}(0)=\left\{\left(0, w_{1}^{0}\right), \ldots,\left(0, w_{s}^{0}\right)\right\}$. Тогда росток $C_{\varepsilon_{1}}$ над точкой 0 кривой $C$ распадается в несвязное объединение

$$
C_{\varepsilon_{1}}=\bigsqcup_{i=1}^{s} C_{\varepsilon_{1}, i}
$$

$W$-подготовленных ростков в точках, имеюших кратности особенностей $k_{i}, 1 \leqslant$ $i \leqslant s, k_{1}+\cdots+k_{s}=m$, с центрами в точках $o_{i}=\left(0, w_{i}\right), 1 \leqslant i \leqslant s$. Пусть $k_{1}, \ldots, k_{t} \geqslant 2$ и $k_{t+1}=\cdots=k_{s}=1$. Далее необходимо выбрать подходяший полидиск для каждой ветви ростка. Для этого выберем $\varepsilon_{3}>0$ и $\varepsilon_{1} \ll \varepsilon_{3}$ так, что каждый росток $C_{\varepsilon_{1}, i}$ содержится в $D_{1} \times E_{i}$, где $E_{i}=\left\{\left|w-w_{i}^{0}\right|<\varepsilon_{3}\right\}$ и $E_{i} \cap E_{j}=\varnothing$ для $i \neq j$. Положим $K_{i}=K_{i}(u)=\left\{w_{i, 1}, \ldots, w_{i, k_{i}}\right\}=D_{2, u} \cap C_{\varepsilon_{1}, i}$ и $E_{i, u}=\left(D_{1} \times E_{i}\right) \cap D_{2, u}$. Вложения $\left(E_{i}, K_{i}\right) \subset\left(D_{2, u}, K\right)$ определяют вложения $\eta_{i}: B_{k_{i}}\left[E_{i, u}, K_{i}\right] \subset B_{m}\left[D_{2, u}, K\right]$ так, что

$$
\tilde{b}_{\left(C_{\varepsilon_{1}}, 0\right)}=\prod_{i=1}^{t} b_{\left(C_{\varepsilon_{1}, i}, o_{i}\right)} \subset B_{m} .
$$

Как и в п. 2.1 , выберем в каждой группе $B_{k_{i}}\left[E_{i, u}, K_{i}\right]$ в качестве стандартных образуюших некоторые полуповороты. Тогда каждое разложение $b_{\left(C_{\varepsilon_{1}, i}, o_{i}\right)}$, будучи отождествленным с его образом $\nu\left(b_{\left(\varepsilon_{\varepsilon_{1}, i}, o_{i}\right)}\right) \in B_{k_{i}}$, становится стандартной формой брэйд-монодромии ростка $C_{\varepsilon_{1}, i}$ относительно pr. Объединение образов при отображениях $\eta_{i}$ этих образуюших может быть расширено до множества стандартных полуповоротов, порождающих группу $B_{m}=B_{m}\left[D_{2, u}, K\right]$.

Таким образом, получаем топологическую монодромию

$$
b_{\left(C_{\varepsilon_{1}}, 0\right)}=b_{\left(C_{\varepsilon_{1}, 1}, o_{1}\right)} \cdot \ldots \cdot b_{\left(C_{\varepsilon_{1}, t}, o_{t}\right)} \in T
$$

(определение $T$ см. в п. 1.3) и называем ее стандартной формой брэйд-монодромии ростка кривой $C$ над точкой 0 .

Описанная вьше конструкция зависит только от нумерации точек $p_{\mid C}^{-1}(0)=$ $\left\{\left(0, w_{1}^{0}\right), \ldots,\left(0, w_{s}^{0}\right)\right\}$ и расширения объединения образов образуюших при отображениях $\eta_{i}$ до множества образующих группы $B_{m}=B_{m}\left[D_{2, u}, K\right]$. Поэтому определение стандартных форм брэйд-монодромии является однозначным с точностью до сопряжения.

Из приведенной вьше конструкции следует, что

$$
\alpha\left(b_{\left(C_{\varepsilon_{1}}, 0\right)}\right)=\tilde{b}_{\left(C_{\varepsilon_{1}}, 0\right)} .
$$

В заключение этого пункта рассмотрим хорошо известное элементарное преобразование, заменяющее росток кривой над точкой на росток особенности. Именно, с ростком кривой $C$ над точкой 0 , заданным уравнением (2.2), можно связать другой росток $\bar{C}_{\varepsilon_{1}}$, заданный уравнением

$$
w^{m}+\sum_{i=1}^{m} z^{i} q_{i}(z) w^{m-i}=0
$$

и имеющий уже только одну точку над точкой 0; назовем его ассоциированной особенностью кривой $C$ над точкой 0 . 
ЗАмЕчАнИЕ 2.1. Геометрический смысл ассоциированной особенности состоит в следуюшем. Можно рассмотреть $\mathbb{C}^{2}$ с координатами $(z, w)$ как аффинную карту некоторой линейчатой поверхности $\mathrm{pr}: \Sigma_{N} \rightarrow \mathbb{P}^{1}, N>0$, не пересекающуюся с исключительным сечением $E_{N}$ поверхности $\Sigma_{N}$, а $C$-как алгебраическую кривую, содержащуюся в этой карте поверхности $\Sigma_{N}$. Сделаем элементарное преобразование $\tau: \Sigma_{N} \rightarrow \Sigma_{N+1}$ с центром в точке пересечения $E_{N}$ со слоем $F_{0}$ над точкой $z=0$. Тогда уравнение (2.3) есть уравнение образа $\tau(C) \subset \Sigma_{N+1}$ в соответствующей карте поверхности $\Sigma_{N+1}$. Ассоциированная особенность определяет однозначно росток кривой $C$ (чтобы получить его, достаточно проделать обратное преобразование $\left.\tau^{-1}\right)$.

Следующее утверждение связывает между собой стандартную форму брэйдмонодромии ростка кривой с брэйд-монодромией ассоциированной с ней особенности. Поскольку оно в статье не используется, мы поместили его доказательство в конец настояшего параграфа.

УТВЕРЖДЕНИЕ 2.1. Пусть $\left(C_{\varepsilon_{1}}, 0\right)$ - росток алгебраической кривой степени $m$ над точкой 0 и $\left(\bar{C}_{\varepsilon_{1}}, o\right)$ - росток ассоциированной с ней особенности. Тогда

$$
b_{\left(\bar{C}_{\varepsilon_{1}}, o\right)}=\alpha\left(b_{\left(C_{\varepsilon_{1}}, 0\right)}\right) \Delta_{m}^{2} .
$$

ЗАмЕчАНИЕ 2.2. Поскольку росток кривой определяется своей ассоциированной особенностью, то из утверждения 2.1 вытекает, что два ростка $\left(C_{1, \varepsilon_{1}}, 0\right)$ и $\left(C_{2, \varepsilon_{1}}, 0\right)$ топологически эквивалентны тогда и только тогда, когда $\alpha\left(b_{\left(C_{1, \varepsilon_{1}}, 0\right)}\right)=$ $\alpha\left(b_{\left(C_{2, \varepsilon_{1}}, 0\right)}\right)$. Тем самым, для определения разложения брэйд-монодромии алгебраической кривой на множители (см. ниже), достаточно полугрупп разложений на множители первого уровня. Однако в $\S 3$ мы расширим исследуемый нами класс алгебраических кривых до класса топологических кривых Гурвища. Разложение брэйд-монодромии на множители топологической кривой Гурвища требует уже рассмотрения полугрупп разложения второго уровня. Поэтому, в целях обшности изложения, мы также определяем разложение брэйд-монодромии алгебраической кривой на множители как элемент некоторой полугруппы разложения на множители второго уровня над группой кос.

2.3. Разложение на множители брэйд-монодромии над диском. Здесь, как и в п. 2.2, рассмотрим многочлен $P(z, w)=w^{m}+\sum_{i=1}^{m} q_{i}(z) w^{m-i} \in \mathbb{C}[z, w]$, не имеюший кратных множителей, и кривую $C$ в $\mathbb{C}^{2}$, заданную уравнением $P(z, w)=0$.

Выберем некоторое $r>0$ так, что критические значения отображения $\left.\mathrm{pr}\right|_{C}$ не лежат на границе диска $D_{1}(r)$. Обозначим через $z_{1}, \ldots, z_{n} \in D_{1}(r)$ критические значения проекции $\left.\mathrm{pr}\right|_{C}$, лежашие в $D_{1}(r)$. Выберем положительное $\varepsilon \ll 1$ так, чтобы диски $D_{1, i}(\varepsilon)=\left\{z \in \mathbb{C}|| z-z_{i} \mid<\varepsilon\right\}, i=1, \ldots, n$, не пересекались друг с другом. Зафиксируем некоторые точки $u_{i} \in \partial D_{1, i}(\varepsilon), 1 \leqslant i \leqslant n$, и точку $u_{0} \in$ $\partial D_{1}(r)$. Положим $D_{2, u_{i}}=\operatorname{pr}^{-1}\left(u_{i}\right), i=0, \ldots, n$, и $K\left(u_{i}\right)=\left\{w_{i, 1}, \ldots, w_{i, m}\right\}=$ $D_{2, u_{i}} \cap C$. Выберем попарно не пересекаюшиеся простые пути $l_{i} \subset \mathrm{Cl} D_{1}(r) \backslash$ $\bigcup_{1}^{n} D_{1, i}(\varepsilon), i=1, \ldots, n$, с началом в $u_{0}$ и конщами в $u_{i}$ и перенумеруем выбранные точки, если это необходимо, так, чтобы произведение $\gamma_{1} \ldots \gamma_{n}$ петель $\gamma_{i}=l_{i}$ 。 $\partial D_{1, i}(\varepsilon) \circ l_{i}^{-1}$ стало равно $\partial D_{1}(r)$ в $\pi_{1}\left(\mathrm{Cl} D_{1}(r) \backslash\left\{z_{1}, \ldots, z_{n}\right\}, u_{0}\right)$ (как обычно, петля $\partial D$ снабжена ориентацией против часовой стрелки). 
Теперь, как и в п. 2.2, каждой петле $\gamma_{i}, 1 \leqslant i \leqslant n$, можно сопоставить элемент $b_{i} \in T_{\varnothing} \subset T \subset S_{B_{m}}, B_{m}=B_{m}\left[D_{2}, K\left(u_{0}\right)\right]$, где $D_{2}$ - диск большого радиуса в $w$-плоскости. Для каждого $1 \leqslant i \leqslant n$ класс сопряженности элемента $b_{i}$ является стандартной формой ростка кривой $C$ над точкой $z_{i}$. Разложение на множители $b_{1} \cdot \ldots \cdot b_{n} \in \mathscr{T}$ называется разложением на множители брәйд-монодромии многочлена $P(z, w)$, или, что то же самое, кривой $C$ над диском $D_{1}(r)$. Для заданного многочлена $P(z, w)$ разложения на множители брэйдмонодромии над $D_{1}(r)$ совпадают друг с другом с точностью до преобразований Гурвица и одновременного сопряжения (см. [18]), т.е. имеют один и тот же тип разложения на множители в смысле определений из $\S 1$. Обозначим тип разложения на множители брэйд-монодромии многочлена $P(z, w)$ над $D_{1}(r)$ (соответственно, кривой $C$, заданной уравнением $P(z, w)=0)$ через $\operatorname{bmt}\left(P(z, w), D_{1}(r)\right)$ (соответственно, $\left.\operatorname{bmt}\left(C, D_{1}(r)\right)\right)$. Если $P(z, w)$ не имеет критических значений вне $D_{1}(r)$, то будем говорить просто о разложении на множители брэйд-монодромии многочлена $P(z, w)$ (или кривой $C$ ), а его тип обозначать $\operatorname{bmt}(P(z, w))($ соответственно, $\operatorname{bmt}(C))$.

Рассмотрим все стандартные формы брэйд-монодромий всех ростков над точкой 0 всех алгебраических кривых степени $m$. Обозначим через $P$ объединение их орбит при действии сопряжением группы $B_{m}$ на $S_{B_{m}}$ и положим $\mathscr{P}=\mathscr{P}_{m}=$ $S\left(S_{B_{m}}, P\right)$. Заметим, что $A_{k} \subset \mathscr{P}$, где $A_{k}$ было определено в п. 1.2 (в частности, элементы, принадлежащие $A_{0}$, соответствуют простым точкам касания кривой $C$ и слоя $z=0$, элементы, принадлежашие $A_{1}$, соответствуют невырожденным двойным точкам (для краткости будем называть такие точки нодами) и элементы, принадлежашие $A_{2}$, соответствуют обыкновенным точкам возврата (для краткости будем называть их обыкновенными каспами)). Поскольку $\mathscr{P}=S\left(S_{B_{m}}, P\right)$ вложено в $\mathscr{T}$, каждое разложение на множители брэйд-монодромии многочлена $P(z, w)$ над диском $D_{1}(r)$ определяет элемент в полугруппе $\mathscr{P}$ и два разложения на множители брэйд-монодромий двух многочленов над диском имеют один и тот же тип тогда и только тогда, когда соответствующие элементы в $\mathscr{P}$ принадлежат одной и той же орбите действия сопряжением группы $B_{m}$ на $\mathscr{P}$. Поэтому будем называть орбиты действия сопряжением группы $B_{m}$ на $\mathscr{P}$ геометрическими типами разложений на множители брәйд-монодромии. Заметим, что $\beta_{S}: \mathscr{P} \rightarrow S_{B_{m}}$ является вложением. Поэтому часто мы не будем делать различий между полугруппой $\mathscr{P}$ и ее образом $\beta_{S}(\mathscr{P})$.

Отметим, что все понятия, введенные в этом пункте, дословно переносятся на любую замкнутую область в $\mathbb{C}$, диффеоморфную замкнутому диску.

2.4. Полиномиальная реализуемость. Пусть $\Sigma_{N}, N \geqslant 1$, - относительно минимальная линейчатая поверхность, pr: $\Sigma_{N} \rightarrow \mathbb{P}^{1}-$ ее линейчатая структура, $F$ - слой проекции pr и $E_{N}$ - исключительное сечение, $E_{N}^{2}=-N$. Выберем точку $\infty \in \mathbb{P}^{1}$ и положим $F_{\infty}=p^{-1}(\infty)$.

Рассмотрим линейную систему

$$
\mathbb{P}=\mathbb{P}_{N, m}=\mathbb{P} H^{0}\left(\Sigma_{N}, \mathscr{O}_{\Sigma_{N}}\left(m E_{N}+m N F\right)\right)
$$

В карте $\Sigma_{N} \backslash\left(F_{\infty} \cup E_{N}\right) \simeq \mathbb{C}^{2}$ можно выбрать координаты $(z, w)$ так, что ограничение проекции $p$ на $\Sigma_{N} \backslash\left(F_{\infty} \cup E_{N}\right)$ совпадет с проекцией $\mathrm{pr}:(z, w) \mapsto z$. Относительно выбранных координат любой элемент $\bar{C} \in \mathbb{P}$ может быть представлен 
уравнением

$$
P(z, w)=0
$$

где $P(z, w)=\sum_{i=0}^{m} q_{i}(z) w^{m-i}$, a $q_{i}(z)=\sum_{j=0}^{i N} a_{i, j} z^{j}$ являются многочленами степеней $\leqslant i N$.

Если $\bar{C}$ является неприводимой кривой, то, поскольку индекс пересечения $\bar{C} E_{N}$ равен нулю, проекция $\operatorname{pr}_{\mid \bar{C}}: \bar{C} \rightarrow \mathbb{C P}^{1}$ является собственным отображением степени $m$. Оно имеет $m(m-1) N$ критических значений (с учетом кратностей), которые могут быть найдены из уравнения

$$
R(z)=0
$$

где $R(z)=R_{P, P_{w}^{\prime}}(z)=\sum r_{k} z^{k}$ - результант многочленов $P(z, w)$ и $P_{w}^{\prime}(z, w)=$ $\frac{\partial}{\partial w} P(z, w), \operatorname{deg} R(z)=m(m-1) N$.

Коэффищиенты $r_{k}$ результанта допускают полиномиальное выражение, зависящее от коэффициентов многочлена $P$. Эти многочлены $r_{k} \in \mathbb{C}\left[a_{0,0}, \ldots, a_{m, m N}\right]$ определяют рациональное отображение

$$
\mathscr{R}: \mathbb{P} \rightarrow \operatorname{Sym}_{m(m-1) N} \mathbb{P}^{1} \simeq \mathbb{P}^{m(m-1) N},
$$

где $\operatorname{Sym}_{m(m-1) N} \mathbb{P}^{1}$ - симметрическое произведение $m(m-1) N$ копий прямой $\mathbb{P}^{1}$. Точкам неопределенности этого отображения соответствуют приводимые кривые $\bar{C} \in \mathbb{P}$ с кратными компонентами, отличными от слоев (регулярность этого отображения в некоторой точке эквивалентна существованию непрерывного продолжения отображения в эту точку, и проверка непрерывности продолжения является непосредственной).

Далее понадобятся следуюшие леммы.

ЛЕмма 2.1. Всякое разложение на множители $b=\operatorname{bmf}(\bar{C}) \in \mathscr{P}$ брэйдмонодромии общей кривой $\bar{C} \in \mathbb{P}$ равно $\left(\delta^{2}\right)^{N}$.

ДокАЗАТЕльство. По лемме $1.2\left(\delta^{2}\right)^{N}$ является единственным элементом в своей орбите при действии сопряжением группы $B_{m}$. Поэтому достаточно показать, что $\left(\delta^{2}\right)^{N}$ равна некоторому разложению брэйд-монодромии на множители. Это равенство хорошо известно в случае $N=1$ (см., например, [15]). Покажем, как вывести общее утверждение из этого частного случая.

Выберем точки $z_{1}, \ldots, z_{N}$, не совпадаюшие с точкой 0 . Рассмотрим кривую $\bar{C}_{0} \in \mathbb{P}$, заданную уравнением

$$
\prod_{j=1}^{m}\left(w z_{2} \ldots z_{N}+(-1)^{N} a_{j}\left(z-z_{1}\right) \ldots\left(z-z_{N}\right)\right)=0, \quad a_{i} \neq a_{j} \text { при } i \neq j
$$

и общую кривую $\bar{C} \in \mathbb{P}$, достаточно близкую к $\bar{C}_{0}$ (т.е. кривую, лежащую в пересечении небольшой топологической окрестности кривой $\bar{C}_{0}$ с множеством кривых $C \in \mathbb{P}$, для которых проекция $\operatorname{pr}_{\mid C}: C \rightarrow \mathbb{C P}^{1}$ имеет только простые критические точки и различные критические значения). Множество критических значений проекции $\operatorname{pr}_{\mid \bar{C}}$ распадается на $N$ подмножеств $\left\{z_{k, 1}, \ldots, z_{k, m(m-1)}\right\}, k=1, \ldots, N$, лежащих в малых дисках $D_{1, k}(\varepsilon)=\left\{\left|z-z_{k}\right|<\varepsilon\right\}, 0<\varepsilon \ll 1$, не пересекающихся друг с другом. Соответственно, $b=b_{1} \cdot \ldots \cdot b_{N}$, где $b_{k}$ - разложение брэйдмонодромии кривой $\bar{C}$ над $D_{1, k}(\varepsilon)$ на множители. 
Покажем, что $b_{1}=\delta^{2}$ (доказательство того, что $b_{2}=\cdots=b_{N}=\delta^{2}$, аналогично). Определим путь $\bar{C}_{0}(t), 0 \leqslant t \leqslant 1$, в $\mathbb{P}$ уравнениями

$$
\begin{gathered}
\prod_{j=1}^{m}\left(w z_{2}(t) \ldots z_{N}(t)+(-1)^{N} a_{j}\left(z-z_{1}\right)\left(z-z_{2}(t)\right) \ldots\left(z-z_{N}(t)\right)\right)=0, \\
z_{j}(t) \rightarrow \infty \text { при } t \rightarrow 1, \quad z_{j}(0)=z_{j} .
\end{gathered}
$$

Он соединяет кривую $\bar{C}_{0}(0)=\bar{C}_{0}$ с кривой $\bar{C}_{0}(1)$, заданной уравнением

$$
\prod_{j=1}^{m}\left(w-a_{j}\left(z-z_{1}\right)\right)=0 .
$$

Поскольку $\mathscr{R}$ является регулярным отображением в каждой точке пути $\bar{C}_{0}(t)$, можно найти достаточно близкий к нему путь $\bar{C}(t)$ такой, что для каждого $t \neq 1$ проекция $\operatorname{pr}: \bar{C}(t) \rightarrow \mathbb{P}^{1}$ имеет только простые критические точки с несовпадающими критическими значениями, $\bar{C}(0)=\bar{C}, \bar{C}(1)$ задана полиномиальным уравнением $\sum_{i+j=m} a_{i, j}\left(z-z_{1}\right)^{i} w^{j}=0$ и для каждого $0 \leqslant t<1$ все $m(m-1)$ критических точек кривой $\bar{C}(t)$ лежат в $\left\{\left|z-z_{1}\right|<\varepsilon\right\}$. Отсюда следует, что разложение на множители брэйд-монодромии кривой $\bar{C}$ над $D_{1}(\varepsilon)$ совпадает с разложением на множители брэйд-монодромии кривой $\bar{C}(1)$ над $D_{1}(\varepsilon)$, которое равно $\delta^{2}$ (случай $N=1$ ).

ЛЕмма 2.2. Пусть кривая $\bar{C}_{0} \in \mathbb{P}$ имеет обыкновенную особенность кратности $m$ в точке $z=0, \quad w=0$, т.е. уравнение кривой $\bar{C}_{0}$ имеет вид $P_{\geqslant m}(z, w)=\sum_{i+j \geqslant m} a_{i, j} z^{i} w^{j}=0$, где многочлен $\sum_{i+j=m} a_{i, j} w^{j}=0$ имеет $m$ различных корней. Пусть $Q(z, w) \in \mathbb{P}-$ такой многочлен, что для некоторого $\varepsilon_{0}>0$ и для всех $0<\varepsilon \ll 1$ кривая $\bar{C}_{\varepsilon}$, заданная уравнением

$$
P_{\geqslant m}(z, w)+\varepsilon Q(z, w)=0,
$$

имеет в точности $m(m-1)$ различных критических значений в круге $D_{1}\left(\varepsilon_{0}\right)=$ $\left\{|z|<\varepsilon_{0}\right\}$. Тогда разложение на множители брәйд-монодромии кривой $\bar{C}_{\varepsilon}$ над $D_{1}\left(\varepsilon_{0}\right)$ равно $\delta^{2}$.

ДокАЗАТЕЛЬСтво. Соединим кривую $\bar{C}_{0}$ с кривой $\bar{C}_{1} \in \mathbb{P}$, заданной уравнением $\sum_{i+j=m} a_{i, j} z^{i} w^{j}=0$, с помощью пути $\left\{\bar{C}_{t}\right\}_{0 \leqslant t \leqslant 1}$ в $\mathbb{P}$, где $\bar{C}_{t}$ задана уравнением

$$
P_{t}(z, w)=\sum_{i+j=m} a_{i, j} z^{i} w^{j}+\sum_{i+j>m} a_{i, j}(t) z^{i} w^{j}=0 .
$$

Поскольку старшая однородная форма уравнений является невырожденной для любого $t$, результант $\mathscr{R}\left(\bar{C}_{t}\right)$ имеет корни $z_{1,0}(t)=\cdots=z_{m(m-1), 0}(t)=0$ и $z_{k, 0}(t) \neq 0$ для $k>m(m-1)$. Поэтому можно выбрать такое $\varepsilon_{0}>0$, что $2 \varepsilon_{0}<\left|z_{k, 0}(t)\right|$ для $k>m(m-1)$ и всех $t \in[0,1]$. Для достаточно малого $\varepsilon$ образ $\left\{\mathscr{R}\left(\bar{C}_{t, \varepsilon}\right)\right\}$ пути $\left\{P_{t}(z, w)+\varepsilon Q(z, w)=0\right\}$ лежит в окрестности $V_{\varepsilon_{0}} \subset$ $\operatorname{Sym}_{m(m-1) N} \mathbb{P}^{1}$ пути $\left\{\mathscr{R}\left(\bar{C}_{t}\right)\right\}$,

$$
\begin{array}{r}
V_{\varepsilon_{0}}=\left\{\left(z_{1}, \ldots, z_{N m(m-1)}\right)|| z_{i} \mid<\varepsilon_{0} \quad \text { для } i \leqslant m(m-1)\right. \\
\text { и } \left.\left|z_{i}\right|>\varepsilon_{0} \text { для } i>m(m-1)\right\} .
\end{array}
$$


Изменяя незначительно коэффициенты многочлена $Q(z, w)$, можно предполагать, что для $0<\varepsilon \ll 1$ и любого $0 \leqslant t \leqslant 1$ кривая $\mathscr{R}\left(\bar{C}_{t, \varepsilon}\right)$ принадлежит множеству $V_{\varepsilon_{0}} \cap\left\{z_{i} \neq z_{j}\right.$ для $\left.i, j \leqslant m(m-1), i \neq j\right\}$. При этом предположении все кривые $\bar{C}_{t, \varepsilon}$ имеют над $D_{\varepsilon_{0}}$ одинаковое разложение брэйд-монодромии на множители.

"Лоскутное шитье Виро" (см. [22]) основано исключительно на квазиоднородных заменах координат и, следовательно, сохраняет брэйд-монодромию. Поэтому, чтобы закончить доказательство, осталось заменить $\bar{C}_{t, \varepsilon}$ обшим многочленом, полученным с помощью "сшивания" общих многочленов

$$
\sum_{i+j<m} a_{i, j}^{\prime} z^{i} w^{j}+\sum_{i+j=m} a_{i, j} z^{i} w^{j}, \quad \sum_{i+j=m} a_{i, j} z^{i} w^{j}+\sum_{i+j>m} a_{i, j}^{\prime} z^{i} w^{j},
$$

близких к $\bar{C}_{1}$, и применить лемму 2.1 (случай $N=1$ ).

ЛЕМма 2.3. Пусть $\bar{b}$ - разложение на множстели брәйд-монодромии аффинной части $C$ проективной кривой $\bar{C} \in \mathbb{P}_{N_{1}, m}$ над диском $D_{1}(r), \quad r \gg 1$. Тогда существует константа $M=M_{C} \in \mathbb{N}$ такая, что для любого $N_{2} \geqslant M$ существует проективная кривая $\widetilde{C}_{1} \in \mathbb{P}_{N_{1}+N_{2}, m}$ такая, что $\tilde{b}=\bar{b} \cdot\left(\delta^{2}\right)^{N_{2}}$ является разложением на множители брэйд-монодромии ее аффинной части $C_{1}$ над диском $D_{1}\left(r_{1}\right), r_{1} \gg r, u \bar{b}$ является разложением на множители брәйд-монодромии кривой $C_{1}$ над диском $D_{1}(r)$.

ДоказАТельство. Обозначим через $z_{1}, \ldots, z_{k}$ критические значения проекции $\operatorname{pr}_{\mid C}$ и через $\left(z_{i}, w_{i, 1}\right), \ldots,\left(z_{i}, w_{i, t_{i}}\right)$ - критические точки над $z=z_{i}$. Сопоставим ростку $\left(C_{i, j},\left(z_{i}, w_{i, j}\right)\right)$ кривой $C$ в точке $\left(z_{i}, w_{i, j}\right)$ число $M_{i, j}$, определение которого было дано в п. 2.1. Положим $M_{i}=\max _{j} M_{i, j}$ и $M_{C}=\sum_{i} M_{i}$. Выберем точки $z_{k+i}, i=1, \ldots, N_{2}$, так, что $\left|z_{k+i}\right| \gg r$, и сделаем $N_{2}>M_{C}$ элементарных преобразований с центрами в точках пересечения слоев $z=z_{k+i}, 1 \leqslant i \leqslant N_{2}$, с исключительным сечением поверхности $\Sigma_{N_{1}+i-1}, i=1, \ldots, N_{2}$. Обозначим через $\widetilde{C} \subset \Sigma_{N_{1}+N_{2}}$ собственньй прообраз кривой $\bar{C}$ при композиции $\tau: \Sigma_{N_{1}} \rightarrow \Sigma_{N_{1}+N_{2}}$ этих преобразований, и пусть

$$
\widetilde{P}(z, w)=w^{m}+\sum_{i=1}^{m} q_{i}(z) w^{m-i}=0
$$

является уравнением ее аффинной части. Единственными критическими значениями проекции $\operatorname{pr}_{\mid \widetilde{C}}$ являются точки $z_{1}, \ldots, z_{k}, z_{k+1}, \ldots, z_{k+N_{2}}$. Над $z=z_{k+i}$ лежит только одна точка кривой $\widetilde{C}$, и в этой точке кривая $\widetilde{C}$ эквивалентна (относительно pr) кривой, заданной уравнением

$$
\prod_{i=1}^{m}\left(w-c_{i}\left(z-z_{k+i}\right)\right)=0
$$

Над $D_{1}(r)$ разложение брэйд-монодромии кривой $\widetilde{C}$ на множители совпадает с разложением на множители брэйд-монодромии кривой $C$, так как центры преобразования $\tau$ лежат над $z_{k+i}, 1 \leqslant i \leqslant N_{2}$, и $\left|z_{k+i}\right| \gg r$. По выбору констант $M_{j}$ и в силу леммы 2.2 кривая $C_{\bar{\varepsilon}}$, заданная уравнением

$$
\widetilde{P}(z, w)+\prod_{i=1}^{k}\left(z-z_{i}\right)^{M_{i}}\left(\sum_{j=0}^{m-1} \varepsilon_{j} w^{j}\right)=0
$$


где все $\varepsilon_{j}$ выбраны общими и достаточно близкими к нулю, имеет $\tilde{b}=\bar{b} \cdot\left(\delta^{2}\right)^{N_{2}}$ своим разложением брэйд-монодромии на множители над $D_{1}\left(r_{1}\right), r_{1} \gg r$. Действительно, по теореме Бертини кривые $C_{\bar{\varepsilon}}$ являются неособыми над $\mathbb{P}^{1} \backslash\left\{z_{1}, \ldots, z_{k}\right\}$ для почти всех $\bar{\varepsilon}$. Более того, если для некоторого $\bar{\varepsilon}$ слой $F$ над точкой, лежащей около некоторой точки $z_{k+i}$, касается кривой $C_{\bar{\varepsilon}}$ с кратностью больше двух в некоторой точке, то можно выбрать $\bar{\varepsilon}_{1}$, близкое к $\bar{\varepsilon}$ и такое, что $C_{\bar{\varepsilon}_{1}}$ и $F$ имеют только простейшую точку касания. Поэтому существует такое $\varepsilon_{0}$, что для почти всех $\bar{\varepsilon}$, достаточно близких к нулю, ровно $m(m-1)$ различных критических значений проекции $\mathrm{pr}: C_{\bar{\varepsilon}} \rightarrow \mathbb{P}^{1}$ лежат в каждой окрестности $\left\{\left|z-z_{k+i}\right|<\varepsilon_{0}\right\}$.

ЛЕмма 2.4. Если $b \in \mathscr{P}$ является разложением на множители брэйд-монодромии над диском $D$ аффинной кривой $P(z, w)=w^{m}+\sum_{i=1}^{m} p_{i}(z) w^{m-i}=0$ с коэффициентами $p_{i}(z)=\sum_{j=0}^{i N} a_{i, j} z^{j}, N \geqslant 1$, то существует такая аффинная кривая $Q(z, w)=w^{m}+\sum_{i=1}^{m} q_{i}(z) w^{m-i}=0$, что:

(i) $q_{i}(z)=\sum_{j=0}^{i N} b_{i, j} z^{j}$

(ii) многочлен $w^{m}+\sum_{i=1}^{m} b_{i, i N} w^{m-i}$ имеет m различных корней;

(iii) все критические точки многочлена $Q(z, w)$, лежсащие над $\mathbb{C} \backslash D$, являются невырожденными; в частности, кривая $C$, заданная уравнением $Q(z, w)=0$, является неособой над $\mathbb{C} \backslash D ;$

(iv) над диском D разложение брәйд-монодромии кривой $С$ на множители равно $b$.

ДоКАЗАТЕЛЬСТво. Выполнения свойства (ii) можно достичь обшим выбором бесконечно удаленного слоя. После этого достаточно применить несколько раз к уравнению

$$
Q(z, w)+\prod_{i=1}^{k}\left(z-z_{i}\right)^{M_{i}}\left(\sum_{j=0}^{m-1} \varepsilon_{j} w^{j}\right)=0
$$

ту же корректирующую процедуру, что и в конце доказательства леммы 2.3.

Теорема 2.1. Для любого $b \in \mathscr{P}$ существуют иисло $N \in \mathbb{N}$ и многочлен $P(z, w)=w^{m}+\sum_{i=1}^{m} q_{i}(z) w^{m-i}$ maкие, что:

(i) $q_{i}(z)=\sum_{j=0}^{i N} a_{i, j} z^{j}$;

(ii) многочлен $w^{m}+\sum_{i=1}^{m} a_{i, i N} w^{m-i}$ имеет $m$ различных корней;

(iii) все критические точки многочлена $P(z, w)$, лежащие над дополнением $\mathbb{C} \backslash D_{1}(1) \kappa$ диску $D_{1}(1)=\{z \in \mathbb{C}|| z \mid<1\}$, являются невырожсденнылми; в частности, кривая $C$, заданная уравнением $P(z, w)=0$, неособа над $\mathbb{C} \backslash D_{1}(1)$;

(iv) $b$ является разложением на множители брэйд-монодромии многочлена $P(z, w)$ над диском $D_{1}(1)$.

ДокАЗАтЕльство. Пусть $b=\rho\left(g_{1}^{-1}\right)\left(b_{1}\right) \cdot \ldots \cdot \rho\left(g_{n}^{-1}\right)\left(b_{n}\right)$, где $b_{j} \in T-$ стандартные формы брэйд-монодромий алгебраических ростков $C_{j}$ над точками $z=z_{j},\left|z_{j}\right|<1$, и пусть

$$
w^{m}+\sum_{i=1}^{m} q_{j, i}\left(z-z_{j}\right) w^{m-i}=0
$$


является уравнением ростка $C_{j}$ над $z=z_{j}$ и

$$
q_{j}(z, w)=0
$$

является уравнением ассоциированной с $C_{j}$ особенности $\bar{C}_{j}$, где

$$
q_{j}(z, w)=w^{m}+\sum_{i=1}^{m}\left(z-z_{j}\right)^{i} q_{j, i}\left(z-z_{j}\right) w^{m-i}
$$

ПРЕДЛОЖЕНИЕ 2.1. Существует полином $Q(z, w)=w^{m}+\sum_{i=1}^{m} \widetilde{q}_{i}(z) w^{m-i}$, имеющий в каждой точке $\left(z_{j}, 0\right)$ особенность того же типа, что $и \bar{C}_{j}, u$ удовлетворяющий условиям (i)-(iii) теоремы 2.1 при некотором $N$.

ДокАЗАТЕльство. Выберем такую константу $M$, что $M>M_{\left(\bar{C}_{j}, z_{j}\right)}$ для всех $j=1, \ldots, n$, где $M_{\left(\bar{C}_{j}, z_{j}\right)}$ - константа, определенная в п. 2.1 .

Сначала построим многочлен $\bar{Q}(z, w)$ степени $\operatorname{deg}_{w} \bar{Q}=m$, имеющий особенности в $\left(z_{j}, 0\right), j=1, \ldots, n$, того же типа, что и $\left(\bar{C}_{j}, z_{j}\right)$. Многочлен $\bar{Q}(z, w)$ будет построен за $n$ шагов. Положим $\bar{Q}_{1}(z, w)=q_{1}(z, w)$. Предположим, что мы построили многочлен $\bar{Q}_{k}(z, w)$ степени $\operatorname{deg}_{w} \bar{Q}_{k}=m$, имеющий в точках $\left(z_{j}, 0\right)$, $j=1, \ldots, k$, особенности того же типа, что и $\left(\bar{C}_{j}, z_{j}\right)$. Рассмотрим многочлен $q_{z_{1}, \ldots, z_{k}}(z)=\left(\left(z-z_{1}\right) \ldots\left(z-z_{k}\right)\right)^{M}$. Имеем $q_{z_{1}, \ldots, z_{k}}\left(z_{k+1}\right) \neq 0$. Следовательно, можно найти такие многочлены $p_{i}(z)$, что многочлен

$$
\bar{Q}_{k+1}(z, w)=\bar{Q}_{k}(z, w)+\sum_{i=1}^{m} q_{z_{1}, \ldots, z_{k}}(z) p_{i}(z) w^{m-i}
$$

имеет те же самые особенности в точках $\left(z_{j}, 0\right), j=1, \ldots, k+1$, что и $\left(\bar{C}_{j}, z_{j}\right)$. Действительно, $\bar{Q}_{k+1}(z, w)$ имеет те же особенности, что и $\left(\bar{C}_{j}, z_{j}\right)$ для $j=1, \ldots, k$, при любом выборе многочленов $p_{i}$, так как $M$ - достаточно большая константа, и при выборе подходящих коэффициентов для многочленов $p_{i}(z)$ многочлен $\bar{Q}_{k+1}(z, w)$ будет иметь тот же тип особенности в точке $\left(z_{k+1}, 0\right)$, что и $\left(\bar{C}_{k+1}, z_{k+1}\right)$. Для завершения доказательства предложения осталось применить лемму 2.4 .

Учитывая замечание 2.1, из предложения 2.1 получаем, что сушествует многочлен $Q(z, w)$, удовлетворяющий условиям (i)-(iii) теоремы 2.1 при некотором $N=N_{1}$ и такой, что множество критических значений проекции $\left.\operatorname{pr}\right|_{Q(z, w)=0}$, лежащих в $D_{1}(1)$, совпадает с $\left\{z_{1}, \ldots, z_{n}\right\}$ и над $z=z_{j}, j=1, \ldots, n$, росток кривой $C$, заданной уравнением $Q(z, w)=0$, имеет тот же топологический тип, что и $\left(C_{j}, z_{j}\right)$. Поэтому над диском $D_{1}(r), r \gg 1$, разложение брэйд-монодромии многочлена $Q(z, w)$ на множители равно

$$
\bar{b}=\rho\left(\bar{g}_{1}^{-1}\right)\left(b_{1}\right) \cdot \ldots \cdot \rho\left(\bar{g}_{n}^{-1}\right)\left(b_{n}\right) \cdot s
$$

где $s \in S_{A_{0}}$. Кроме того, из условий (i)-(iii) теоремы 2.1 следует, что $C \subset \bar{C}$ является аффинной частью проективной кривой $\bar{C} \in \Sigma_{N_{1}}$ такой, что все особые точки кривой $\bar{C}$ принадлежат $C$.

Из теоремы 1.1 , предложения 2.1 и леммы 2.3 следует, что существуют константа $N_{2} \gg 1$ и многочлен $\bar{P}(z, w)$ степени $\operatorname{deg}_{w}=m$, брэйд-монодромия которого 
над диском $D_{1}(r), r \gg 1$, имеет разложение на множители $b \cdot s \cdot\left(\delta^{2}\right)^{N_{2}}$, где $s$ - некотоьй элемент, принадлежащий $S_{A_{0}}$. Следовательно, существует такая область $U \subset \mathbb{C}$, диффеоморфная диску $D_{1}(1)$, что $\bar{P}(z, w)$ имеет над $U$ брэйд-монодромию, разложение на множители которой равно $b$. Сушествует аналитический изоморфизм между $U$ и $D_{1}(1)$. Следовательно, сушествует многочлен $\widetilde{P}(z, w)=w^{m}+$ $\sum_{i=1}^{m} q_{i}(z) w^{m-i}$ с голоморфными в $D_{1}(1)$ коэффищиентами $q_{i}(z)$ такой, что над $D_{1}(1)$ брэйд-монодромия многочлена $\widetilde{P}(z, w)$ имеет разложение на множители, равное $b$. Теперь осталось только аппроксимировать голоморфные функции $q_{i}(z)$ многочленами, струи которых над лежащими в $D_{1}(1)$ критическими значениями проекции $p$ равны соответствуюшим струям (достаточно большого порядка) функций $q_{i}(z)$, и затем применить лемму 2.4 .

ЗАмечАниЕ 2.3. Для заданного $b \in \mathscr{P}$ такого, что $\beta(b)=\Delta^{2}$, рассмотрим многочлен

$$
P(z, w)=w^{m}+\sum_{i=1}^{m} q_{i}(z) w^{m-i},
$$

сушествование которого доказано в теореме 2.1. Как следует из предложения 3.1 (см. ниже), даже в случае особенностей типов $A_{n}, n \leqslant 2$ (простые касательные, ноды и каспы), не существует оценки сверху на степени многочленов $q_{i}(z, w)$, зависящей только от топологических типов критических точек (относительно проекции $\mathrm{pr}$ ) многочлена $P(z, w)=0$. С другой стороны, как это следует из доказательства теоремы 2.1, такая эффективная оценка степени $\operatorname{deg}_{z} \bar{P}$ по $z$ существует при условии, что мы рассматриваем разложение брэйд-монодромии на множители, не фиксируя область.

ЗАмЕчАниЕ 2.4. Случай $N=1$ покрьвает случай алгебраических кривых в $\mathbb{C P}^{2}$. В последнем случае для того, чтобы определить разложение брэйд-монодромии на множители, выбирается точка, не лежащая на кривой, и раздутие этой точки приводит случай плоских кривых к случаю кривых в $\Sigma_{1}$.

ДокАЗАТЕЛЬСТво УТВЕРЖДЕНИЯ 2.1. Реализуем росток $\left(C_{\varepsilon_{1}}, 0\right)$ алгебраической кривой над точкой 0 как росток некоторой проективной кривой $\widetilde{C} \subset \Sigma_{N}$. Пусть $b(\widetilde{\widetilde{C}})$ - разложение ее брэйд-монодромии на множители. Тогда $b(\widetilde{C})=$ $b_{\left(\varepsilon_{\varepsilon_{1}}, 0\right)} \cdot \tilde{b}$, где $\tilde{b} \in \mathscr{P}$ - произведение брэйд-монодромий по всем остальным (кроме точки 0$)$ критическим значениям проекции pr. Имеем

$$
\beta(b(\widetilde{C}))=\beta\left(b_{\left(C_{\varepsilon_{1}}, 0\right)}\right) \beta(\tilde{b})=\Delta_{m}^{2 N} .
$$

Сделаем элеметарное преобразование $\tau$ с центром в $F_{0} \cap E_{\infty} . \quad$ Кривая $\tau^{-1}(\widetilde{C})$ имеет те же критические значения, что и $\widetilde{C}$, а ее брэйд-монодромия будет иметь разложение на множетели $b\left(\tau^{-1}(\widetilde{C})\right)=b_{\left(\bar{C}_{\varepsilon_{1}}, o\right)} \cdot \tilde{b}$, причем

$$
\beta\left(b\left(\tau^{-1}(\widetilde{C})\right)\right)=\beta\left(b_{\left(\bar{C}_{\varepsilon_{1}}, o\right)}\right) \beta(\tilde{b})=\Delta_{m}^{2(N+1)}
$$

Следовательно, $\beta\left(b_{\left(\bar{C}_{\varepsilon_{1}}, 0\right)}\right)=\beta\left(b_{\left(C_{\varepsilon_{1}}, o\right)}\right) \Delta_{m}^{2}$ и из сделанных в п. 1.3 отождествлений вытекают равенства $\beta\left(b_{\left(\bar{C}_{\varepsilon_{1}}, o\right)}\right)=b_{\left(\bar{C}_{\varepsilon_{1}}, o\right)}$ и $\beta\left(b_{\left(C_{\varepsilon_{1}}, 0\right)}\right)=\alpha\left(b_{\left(C_{\varepsilon_{1}}, 0\right)}\right)$. 


\section{§3. Разложения на множители брэйд-монодромии кривых Гурвица}

3.1. Кривые Гурвица. Как и в $\S 2$, пусть $\Sigma_{N}$ - относительно минимальная рациональная линейчатая поверхность, $N \geqslant 1, \operatorname{pr}: \Sigma_{N} \rightarrow \mathbb{P}^{1}$-ее линейчатая структура, $F$ - слой проекции рr и $E_{N}$ - исключительное сечение, $E_{N}^{2}=-N$.

ОПРЕДЕЛЕНИЕ 3.1. Образ $\bar{H}=f(\mathfrak{S}) \subset \Sigma_{N}$ гладкого отображения $f: \mathfrak{S} \rightarrow$ $\Sigma_{N} \backslash E_{N}$ ориентированной замкнутой вешественной поверхности $\mathfrak{S}$ называется кривой Гурвица $\left(\right.$ в $\left.\Sigma_{N}\right)$ степени $m$, если существует конечное подмножество $Z \subset$ $\bar{H}$ такое, что:

(i) $f$ является вложением поверхности $\mathfrak{S} \backslash f^{-1}(Z)$ и для любого $s \notin Z$ образ $\bar{H}$ и слой $F_{\operatorname{pr}(s)}$ проекции $\mathrm{pr}$ пересекаются в $s$ трансверсально с положительным индексом пересечения;

(ii) для каждого $s \in Z$ сушествует такая окрестность $U \subset \Sigma_{N}$ точки $s$, для которой $\bar{H} \cap U$ является комплексно-аналитической кривой и комплексная ориентация на $\bar{H} \cap U \backslash\{s\}$ совпадает с ориентацией, индуцированной ориентацией на $\mathfrak{S}$ с помощью отображения $f$;

(iii) ограничение проекции pr на $\bar{H}$ является конечным отображением степени $m$.

Для каждой кривой Гурвица $\bar{H}$ существует единственное минимальное множество $Z \subset \bar{H}$, которое удовлетворяет условиям определения 3.1. Обозначим его через $Z(\bar{H})$.

Кривая Гурвица $\bar{H}$ называется каспидальной, если для каждой точки $s \in Z$ существуют окрестность $U$ точки $s$ и локально аналитические координаты $x, y$ в $U$ такие, что:

(iv) проекция $\operatorname{pr}_{\mid U}$ совпадает с отображением $(x, y) \mapsto x$;

(v) кривая $\bar{H} \cap U$ задается уравнением $y^{2}=x^{k}, k \geqslant 1$.

Кривая Гурвища называется обыкновенной каспидальной, если в условии (v) имеет место неравенство $k \leqslant 3$ для всех $s \in Z$, и нодальной, если $k \leqslant 2$.

Поскольку $H \cap E_{N}=\varnothing$, можно определить разложение на множители брэйдмонодромии $b(\bar{H}) \in \mathscr{P}$ кривой $\bar{H}$ точно так же, как и в алгебраическом случае. Для этого зафиксируем слой $F_{\infty}$, пересекаюший трансверсально кривую $\bar{H}$, и рассмотрим $H=\bar{H} \cap \mathbb{C}^{2}$, где $\mathbb{C}^{2}=\Sigma_{N} \backslash\left(E_{N} \cup F_{\infty}\right)$. Выберем $r \gg 1$ такое, что $\operatorname{pr}(Z) \subset D_{1}(r) \subset \mathbb{C}=\mathbb{C P}^{1} \backslash \operatorname{pr} F_{\infty}, Z=Z(\bar{H})$. Обозначим через $z_{1}, \ldots, z_{n}$ элементы множества $\operatorname{pr}(Z)$ и выберем $\varepsilon, 0<\varepsilon \ll 1$, такое, что диски $D_{1, i}(\varepsilon)=\left\{z \in \mathbb{C}|| z-z_{i} \mid<\varepsilon\right\}, i=1, \ldots, n$, не пересекаются друг с другом. Выберем точки $u_{i} \in \partial D_{1, i}(\varepsilon)$ и точку $u_{0} \in \partial D_{1}(r)$. Положим $D_{2, u_{i}}=\operatorname{pr}^{-1}\left(u_{i}\right)$, $i=0, \ldots, n$, и $K\left(u_{i}\right)=\left\{w_{i, 1}, \ldots, w_{i, m}\right\}=D_{2, u_{i}} \cap H$. Выберем попарно не пересекаюшиеся друг с другом простые пути $l_{i} \subset \mathrm{Cl} D_{1}(r) \backslash \bigcup_{1}^{n} D_{1, i}(\varepsilon), i=1, \ldots, n$, с началом в $u_{0}$ и концами в точках $u_{i}$ и перенумеруем точки таким образом, чтобы произведение $\gamma_{1} \ldots \gamma_{n}$ петель $\gamma_{i}=l_{i} \circ \partial D_{1, i}(\varepsilon) \circ l_{i}^{-1}$ было равно элементу $\partial D_{1}(r)$ в $\pi_{1}\left(\mathrm{Cl} D_{1}(r) \backslash\left\{z_{1}, \ldots, z_{n}\right\}, u_{0}\right)$.

Как и в $\S 2$, каждая петля $\gamma_{i}$ определяет элемент $b_{i} \in T \subset S_{B_{m}}$. Разложение на множители $b_{1} \cdot \ldots \cdot b_{n} \in \mathscr{T}$ называется разложением на множители брәйд-монодромии кривой $\bar{H}$. Фактически, каждый множитель $b_{i}$ сопряжен стандартной форме брэйд-монодромии некоторого ростка алгебраической кривой над точкой $z_{i}$. Поэтому $b(\bar{H})=b_{1} \cdot \ldots \cdot b_{n}$ принадлежит полугруппе $\mathscr{P}$ (см. определение $\mathscr{P}$ в п. 2.3). Орбита этого элемента при действии сопряжением группы $B_{m}$ 
на $\mathscr{P}$ назьвается геометрическим типом разложения на множители брәйдмонодромии и обозначается bmt.

ЛЕмма 3.1. Для кривой Гурвица $\bar{H} \subset \Sigma_{N}$ имеем $\beta(b(\bar{H}))=\Delta^{2 N}$.

ДокАЗАТЕЛЬСТво. Над $\mathbb{P}^{1} \backslash D_{1}(r)$ кривая $\bar{H}$ есть объединение $m=\operatorname{deg} \bar{H}$ попарно не пересекающихся друг с другом сечений проекции pr. C помощью изотопии эти сечения могут быть преобразованы в сечения, заданные в аффинных координатах уравнением $w=v_{i} z^{N}, 1 \leqslant i \leqslant m, v_{i} \in \mathbb{C}$. Полученные сечения имеют брэйд-монодромию $\Delta^{2 N}$ над $\partial D_{1}(r)$.

Также непосредственно доказывается и обратное утверждение.

Tеорема 3.1 [17]. Для любого $b=b_{1} \cdot \ldots \cdot b_{n} \in \mathscr{P}$ такого, что $\beta(b)=\Delta^{2 N}$, существует кривая Гурвица $\bar{H} \subset \Sigma_{N}$ с разложением на множители брәйдмонодромии $b(\bar{H})$, равным $b$.

3.2. Гурвицевские изотопии. В определении, которое приведено ниже, мы обобщаем понятие кривой Гурвица. Это обобщение соответствует замене полугруппы $\mathscr{P}$ на $\mathscr{T}$.

ОПРЕДЕЛЕНИЕ 3.2. Образ $\bar{H}=f(\mathfrak{S}) \subset \Sigma_{N}$ непрерывного отображения $f: \mathfrak{S} \rightarrow$ $\Sigma_{N} \backslash E_{N}$ гладкой ориентированной замкнутой вешественной поверхности $\mathfrak{S}$ называется топологической кривой Гурвича $\left(\right.$ в $\left.\Sigma_{N}\right)$ степени $m$, если существует конечное подмножество $Z \subset \bar{H}$ такое, что:

(i) $f$ является гладким вложением поверхности $\mathfrak{S} \backslash f^{-1}(Z)$ и для любой точки $s \notin Z$ образ $\bar{H}$ и слой $F_{\operatorname{pr}(s)}$ проекции $\operatorname{pr}$ пересекаются в $s$ трансверсально с положительным индексом пересечения;

(ii) ограничение проекции pr на $\bar{H}$ является конечным отображением степени $m$.

(Мы называем отображение конечным, если прообраз каждой точки состоит из конечного числа точек.)

Как и в случае кривых Гурвища, существует одно и только одно минимальное множество $Z$, которое мы обозначаем через $Z(\bar{H})$. Скажем, что кривая $\bar{H}$ является $Z$-общей (относительно pr), если ограничение проекции pr на $Z(\bar{H})$ является инъекцией.

ОПРЕДЕЛЕниЕ 3.3. Две кривые Гурвица (или две топологические кривые Гурвища) $\bar{H}_{1}$ и $\bar{H}_{2} \subset \Sigma_{N}$ назьваются $H$-изотопнылми , если существует послойная непрерывная изотопия $\phi_{t}: \Sigma_{N} \rightarrow \Sigma_{N}, t \in[0,1]$, гладкая вне слоев $F_{\operatorname{pr}(s)}, s \in Z(\bar{H})$, такая, что:

(i) $\phi_{0}=\mathrm{Id}$;

(ii) $\phi_{t}\left(\bar{H}_{1}\right)$ является кривой Гурвица для каждого $t \in[0,1]$ и $\phi_{t}$ переводит слои проекции $\operatorname{pr}_{\mid \bar{H}_{1}}$ в слои проекции $\operatorname{pr}_{\mid \phi_{t}\left(\bar{H}_{1}\right)}$;

(iii) $\phi_{1}\left(\bar{H}_{1}\right)=\bar{H}_{2}$;

(iv) $\phi_{t}\left(E_{N}\right)=E_{N}$ для всех $t \in[0,1]$.

Если $\bar{H}_{1}$ является топологической кривой Гурвища, то в каждой точке $p \in$ $Z\left(\bar{H}_{1}\right)$ существует хорошо определенный ( $W$-подготовленный) росток $\left(D, H_{1}=\right.$ $\bar{H}_{1} \cap D$, pr) этой кривой в бидиске $D=D_{1} \times D_{2}, D_{1}=D_{1}\left(\varepsilon_{1}\right), D_{2}=D_{2}\left(\varepsilon_{2}\right)$, $0<\varepsilon_{1} \ll \varepsilon_{2}$, с центром в $p$ такой, что ограничение проекции pr на $H_{1}$ является собственным отображением конечной степени. Если $\varepsilon_{1}, \varepsilon_{2}$ достаточно малы, то 
$F_{\operatorname{pr}(p)} \cap H_{1}=p$, упомянутая выше степень не зависит от $\varepsilon_{1}, \varepsilon_{2}$, и так же, как и в алгебраическом случае, линк $\partial D \cap H_{1}$ определяет однозначно с точностью до сопряжения некоторую косу $b \in B_{k}$, где $k$ - степень проекции. Таким образом, можно говорить о $t H$-особенности $\left(D, H_{1}, \mathrm{pr}\right)$ степени $k$, типа $b$ и индекса сплетения $l(b)=l$.

Если задан линк $L \subset \partial D_{1} \times D_{2}$, реализующий косу $b \in B_{k}$, можно связать с ним стандартную каноническую модель топологической особенности типа $b$. Она задается ростком $H=C(L)$,

$$
C(L)=\{(r z, r w) \mid 0 \leqslant r \leqslant 1,(z, w) \in L\} .
$$

Хорошо известно, что если $(D, C)$ - росток $W$-подготовленной аналитической особенности, то росток $(D, C, \mathrm{pr})$ гомеоморфен конической особенности типа $b=$ $\operatorname{pr}^{-1}\left(\partial D_{1} \cap C\right)$.

Каждую $t H$-особенность $\left(D, H_{1}, \mathrm{pr}\right)$ удобно описьвать с помошью потока. Чтобы соответствующие формулы были более прозрачны, положим $\varepsilon_{1}=\varepsilon_{2}=1$ и поместим $H_{1}$ с помощью подходящей, сохраняющей слои $H$-изотопии (подобной гомотетии) внутрь конического тела $|w|<\rho|z|, \quad \rho=1-\varepsilon$. Рассмотрим гладкое отображение $v$ из $D_{1} \backslash\{0\}$ в пространтсво гладких векторных полей на $D_{2}$ такое, что $v(z)(w)=-w$ для всех $|w| \geqslant \rho|z|$. Тогда поток, заданный векторным полем

$$
\frac{d z}{d t}=-z, \quad \frac{d w}{d t}=v(z)(w)
$$

преобразует любую косу $L \subset \partial D_{1}(1) \times D_{2}(\rho)$ в некоторую $t H$-особенность. Обратно, любая $t H$-особенность $\left(D, H_{1}, \mathrm{pr}\right)$ может быть так представлена, если только $H_{1}$ содержится в $|w|<\rho|z|$. Например, стандартная коническая модель $C(L)$ задается парой $(v, \rho)$, где $v(z)(w)=-w$ для всех $z, w$.

УТВЕРЖДЕНИЕ 3.1. Для любой $t H$-особенности $\left(D, H_{1}, \mathrm{pr}\right), \quad D=D_{1} \times D_{2}$, существует H-изотопия (оставляющая неподвижным каждый слой), тождественная над $\partial D$ и преобразующая особенность в ее стандартную коническую модель $C(L), \quad L=H_{1} \cap\left(\partial D_{1} \times D_{2}\right)$.

ДоказАТЕльство. Как и выше, зададим $t H$-особенность $\left(D, H_{1}, \mathrm{pr}\right)$ и ее каноническую модель $C(L)$ с помошью двух потоков: один поток связан с парой $\left(v_{0}, \rho\right)$, а другой - с парой $\left(v_{1}, \rho\right)$. Рассмотрим семейство $t H$-особенностей, заданное парами $\left(v_{t}, \rho\right), v_{t}=t v_{1}+(1-t) v_{0}$, и обозначим через $\Phi_{t, z}$ вложения $\left\{\frac{z}{|z|}\right\} \times D_{2} \rightarrow\{z\} \times D_{2}$, заданные потоком, ассоциированным с $v_{t}$. Чтобы сопроводить построенное семейство $t H$-особенностей объемлющей $H$-изотопией $\phi_{t}$, преобразующей $H_{1}$ в $C(L)$ над $D_{1}$, достаточно взять поток, ассоциированный с вертикальными векторными полями $\frac{d}{d t}\left(\Phi_{t, z} \circ \Phi_{1, z}^{-1}\right)$, продолжая их нулевым полем на все $D_{1} \times D_{2}$ для каждого $t$.

ЗАМЕчАНИЕ 3.1. Фактически, процедура, которую мы использовали в доказательстве утверждения 3.1 , влечет также $H$-изотопию между любыми двумя $t H$-особенностями с одним и тем же линком $L$. Если обе особенности были особыми точками кривых Гурвища, то они в процессе всей изотопии будут оставаться особенностями кривых Гурвица (т.е. алгебраическими особенностями). Однако эта изотопия не обязательно является гладкой в особой точке. 
Определение брэйд-монодромных разложений на множители и их типов дословно переносится со случая кривых Гурвица на случай топологических кривых Гурвица. Отметим только, что если $(D, H, \mathrm{pr})$ является $t H$-особенностью, то в соответствии с утверждением 3.1 она с точностью до $H$-изотопии, тождественной на $\partial D$, определяется однозначно своей замкнутой косой $b=H \cap \operatorname{pr}^{-1}\left(\partial D_{1}\right)$ и, соответственно, ее стандартной tbmf-формой (см. п. 1.3). Следовательно, брэйдмонодромия ростка над точкой топологической кривой Гурвица естественным образом определяется как элемент из $T$, а разложение на множители брэйд-монодромии топологической кривой - как элемент из $\mathscr{T}$. Для разложения на множители брэйд-монодромии топологической кривой Гурвица имеем $\beta(b(\bar{H}))=\Delta^{2 N}$. Обратное утверждение о том, что каждое разложение на множители $b \in \mathscr{T}$ с этим свойством реализуется топологической кривой Гурвища, имеет то же доказательство, что и теорема 3.1.

Группа кос $B_{k}=B\left[D_{2},\left\{w_{1}, \ldots, w_{k}\right\}\right], w_{1}, \ldots, w_{k} \in D_{2}$, естественным образом действует на фундаментальной группе $\pi_{1}=\pi_{1}\left(D_{2} \backslash\left\{w_{1}, \ldots, w_{k}\right\}\right)$. Скажем, что действие элемента $b \in B_{k}$ на $\pi_{1}$ нерасщепляемо, если лишь элементы подгруппы, порожденной в $\pi_{1}$ элементом $\partial D_{2}$, являются неподвижными при действии элемента $b$. Если зафиксированы стандартные образующие $a_{1}, \ldots, a_{k-1}$ группы $B_{k}$, то геометрический базис группы $\pi_{1}\left(D_{2} \backslash\left\{w_{1}, \ldots, w_{k}\right\}\right)$ - это такое множество ее образующих $\left\{x_{1}, \ldots, x_{k}\right\}$, в котором естественное действие группы кос $B_{k}$ задается стандартными формулами, т.е. $a_{i}\left(x_{j}\right)=x_{j}$ при $j \neq i, i+1$ и $a_{i}\left(x_{i+1}\right)=x_{i}$, $a_{i}\left(x_{i}\right)=x_{i} x_{i+1} x_{i}^{-1}$ при $i \leqslant k-1$. Для любого геометрического базиса имеем $\partial D_{2}=x_{1} \ldots x_{k}$.

ЛЕмма 3.2. Пусть $b \in B_{k}$ - нерасщепляемый элемент $u\left\{x_{1}, \ldots, x_{m}\right\}$ геометрический базис группь $\pi_{1}\left(D_{2} \backslash\left\{w_{1}, \ldots, w_{m}\right\}\right)$. Интерпретируем $b$ как әлемент әруппьи $B_{k, 0}=B\left[D_{2},\left\{w_{1}, \ldots, w_{k}\right\}\right] \subset B\left[D_{2},\left\{w_{1}, \ldots, w_{m}\right\}\right]=B_{m} u$ рассмотрим действие на $\pi_{1}=\pi_{1}\left(D_{2} \backslash\left\{w_{1}, \ldots, w_{m}\right\}\right)$, индуцированное әлементом $b$. Тогда подгруппа $F(b)$ неподвижсных әлементов в $\pi_{1}$ относительно действия $b$ порождается әлементом $l=x_{1} \ldots x_{k}$ и әлементами $x_{k+1}, \ldots, x_{m}$.

ДокаЗАТЕЛЬСтво. Очевидно, $l$ и $x_{k+i}$ при $i \geqslant 1$ принадлежат группе $F(b)$. Пусть $g \in F(b)$. Запишем $g$ как приведенное слово, состоящее из букв алфавита $\left\{x_{1}, \ldots, x_{m}\right\}$ и обратных к ним:

$$
g=s_{1} s_{2} \ldots s_{n}
$$

где каждое $s_{i}$ - приведенное слово, не пустое, если $i \neq 1$, состояшее из букв алфавита $\left\{x_{1}, \ldots, x_{k}\right\}$ и обратных к ним, если $i$ - нечетное число, и состоящее из букв алфавита $\left\{x_{k+1}, \ldots, x_{m}\right\}$ и обратных к ним, если $i$ четно. Поскольку такое представление единственно и $g \in F(b)$, то каждый элемент $s_{i}$ принадлежит $F(b)$ и доказательство леммы вытекает из определения нерасшепляемых элементов.

Скажем, что $t H$-особенность $(D, H, \mathrm{pr})$ имеет нерасщепляемый тип, если ее тип $b \in B_{k}$ нерасщепляем. Отметим, что $t H$-особенность нерасшепляемого типа $b$ имеет tbmf-форму $\left(b, \mathbf{1}_{\varnothing}\right)$.

ЛЕмма 3.3. Особенность алгебраической кривой, заданная уравнением $w^{k}=z^{n}$, является нерасщепляемой для всех $n \geqslant 1 u k \geqslant 1$. 
ДокАЗАТЕЛЬСТВо. Тип такой особенности равен $b=\left(a_{1} \ldots a_{k-1}\right)^{n} \in B_{k}$, где $a_{1}, \ldots, a_{k-1}-$ множество стандартных образуюших групшы кос $B_{k}$. Равенство $\Delta_{k}^{2}=\left(a_{1} \ldots a_{k-1}\right)^{k}$ влечет $b^{k}=\Delta_{k}^{2 n}$. С другой стороны, элемент $\Delta_{k}^{2}$ действует как сопряжение на элемент $\partial D_{2} \in \pi_{1}$. Осталось заметить, что централизатор любого элемента $g \neq 1$ в свободной группе совпадает с максимальной циклической подгруппой, содержащей элемент $g$.

Следующий пример показывает, что аналог утверждения 3.1 не может быть распространен на произвольный росток топологической кривой Гурвица над точкой, по крайней мере, если росток состоит из нескольких связных компонент, одна из которых не является особенностью нерасщепляемого типа.

ПримеР 3.1. Пусть $m=3, \operatorname{bmf}\left(H_{1}\right)=\operatorname{bmf}\left(H_{2}\right)=\left(\mathbf{1}, \mathbf{1}_{\{1,2\}}\right), H_{1}$ и $H_{2}-$ конусы врашения (т.е. инвариантны при врашении $\left.(z, w) \mapsto\left(e^{i \phi} z, w\right)\right)$, у ростка $H_{1} \mathrm{~V}$-образная компонента, получающаяся вращением конуса над двумя точками (лежашего в $\{\operatorname{Im} z=0\} \times D_{2}$; эта компонента соответствует элементу $\mathbf{1}_{\{1,2\}}$ ), а другая компонента получена врашением струны, не зацепленной с упомянутым выше конусом, тогда как в $H_{2}$ врашаемая струна зацеплена с одной ветвью (и только с ней) V-образного конуса.

Теорема 3.2. Z-общие топологические кривые Гурвица с особенностями нерасщепляемого типа являются $H$-изотопньци тогда и только тогда, когда имеют одинаковые типы брәйд-монодромии.

ДоКАЗАТЕЛЬСТВО. Необходимость совпадения типов брэйд-монодромий для существования $H$-изотопии является очевидной.

Предположим, что $Z$-общие топологические кривые Гурвища $\bar{H}_{1}, \bar{H}_{2}$ имеют особенности только нерасщепляемого типа, и пусть $\operatorname{bmt}\left(\bar{H}_{1}\right)=\operatorname{bmt}\left(\bar{H}_{2}\right)$. Последнее условие влечет существование $H$-изотопии, которая преобразует $\bar{H}_{1}$ в $\bar{H}_{2}$ всюду, за исключением несвязного объединения открытых множеств, лежащих над малыми дисками с центрами в точках из $\operatorname{pr} Z\left(\bar{H}_{1}\right)$, а сами эти диски в результате изотопии преобразуются в некоторые диски с центрами в $\operatorname{pr} Z\left(\bar{H}_{2}\right)$ (см. [11]). Поэтому остается решить проблему продолжения $H$-изотопии с границы каждого диска на внутренние точки. Здесь предположение о типе особенностей является сушественным.

Итак, предположим, что следы $H_{1}, H_{2}$ кривых $\bar{H}_{1}, \bar{H}_{2}$ над диском $D_{1}=D_{1}(\varepsilon)$ содержатся в $D=D_{1} \times D_{2}(r)$, совпадают над границей диска $D_{1}$ и их брэйдмонодромия $b \in T$ над $\partial D_{1}$ имеет нерасщепляемый тип. Кроме того, предполагаем, что $D_{1}$ содержит только по одной точке из $Z\left(H_{1}\right)$ и из $Z\left(H_{2}\right)$. Не ограничивая общности, можно считать, что $Z\left(H_{1}\right)=Z\left(H_{2}\right)=\{(0,0)\}$. Теперь достаточно построить $H$-изотопию, тождественную на гранище, которая переводит $H_{1}$ в $H_{2}$ по крайней мере над некоторым интервалом, соединяющим некоторую точку, лежащую на границе диска $D_{1}$, с точкой 0 , например, над радиусом $I_{-}=\{\operatorname{Re} z \leqslant 0$, $\operatorname{Im} z=0\} \subset D_{1}$, а затем продолжить ее над некоторой окрестностью точки 0 . Тогда эта $H$-изотопия может быть легко продолжена над оставшейся частью диска $D_{1}$, так как эта часть есть топологический диск, над которым уже нет особых точек.

Пусть $l \geqslant 2$ - индекс сплетения, которьй равен степени особенности, так как $\bar{H}_{i}$ имеет лишь особенности нерасщепляемого типа. Исходя из определения множества $T$, стандартная tbmf-форма особенностей имеет вид $\left(\tilde{b}_{1}, \mathbf{1}_{\varnothing}\right)$, где $b_{1} \in$ 
$B_{l, 0}$ сопряжен элементу $b$, и по определению разложения на множители брэйдмонодромии получаем, что $H_{1}$ и $H_{2}$ распадаются на $k+1=m-l+1$ связных компонент $H_{i, 1}, \ldots, H_{i, k+1}, i=1,2$, и эти разложения на компоненты совпадают над $\partial D_{1}$. Каждая компонента является топологическим конусом; $H_{i, 1}-$ конус над косой $b_{1} \in B_{l, 0}$, а остальные конусы - это конусы над 1-косами $b_{2} \in$ $B_{1, l}, \ldots, b_{k+1} \in B_{1, m-1}$. В соответствии с утверждением 3.1 можно предположить, что $H_{1,1}=H_{2,1}=C(L)$, где $L$ - это след кривой $H_{1,1}=H_{2,1}$ в $\partial D_{1} \times D_{2}(r)$. Кроме того, можно предполагать, что след конуса $C(L)$ над некоторым диском $D_{1}\left(\varepsilon^{\prime}\right), \varepsilon^{\prime} \ll \varepsilon$, содержится в $D_{1}\left(\varepsilon^{\prime}\right) \times \operatorname{Int} D_{2}\left(r^{\prime}\right), r^{\prime} \ll r$, и что $H_{1,2}, \ldots, H_{1, k+1}$ (соответственно, $H_{2,2}, \ldots, H_{2, k+1}$ ) являются различными постоянными сечениями проекции pr над $D_{1}\left(\varepsilon^{\prime}\right)$, заданными уравнениями $w=w_{j}, l+1 \leqslant j \leqslant m$ (соответственно, $\left.w=w_{j}^{\prime}\right)$ и содержашимися в $D_{1}\left(\varepsilon^{\prime}\right) \times A_{2}, A_{2}=\left\{r^{\prime} \leqslant|w| \leqslant r\right\} \subset D_{2}(r)$. С помощью некоторой $H$-изотопии значения $w_{j}\left(\right.$ соответственно, $\left.w_{j}^{\prime}\right)$ этих постоянных сечений могут быть произвольно непрерывно изменены, следуя за произвольной косой, не проходящей через $D_{2}\left(r^{\prime}\right)$. Будем называть такие замены скручиванием-раскручиванием. Отметим, что когда мы получим равенства $w_{j}=w_{j}^{\prime}$ для всех $j \geqslant l+1$, это даст искомое равенство $H_{1}=H_{2}$ над окрестностью точки 0 . Итак, достаточно показать, что сушествует некоторая $H$-изотопия, тождественная на гранище и преобразуюшая $H_{1}$ в $H_{2}$ над $I_{-}$.

Для описания действия элемента $b_{1}$ на $\pi_{1}\left(D_{2}(r) \backslash\left\{w_{1}, \ldots, w_{l}\right\}, w_{0}\right), w_{0} \in \partial D_{2}(r)$, заменим ограничение проекции $\mathrm{pr}_{2}: T \rightarrow D_{2}(r)$ на $T=A_{1} \times D_{2}(r)$, где $A_{1}=\left\{\varepsilon^{\prime} \leqslant\right.$ $|z| \leqslant \varepsilon\} \subset D_{1}$, некоторой проекцией $\mathrm{pr}^{\prime}: T \rightarrow D_{2}(r)$, которая совпадает с $\mathrm{pr}_{2}$ на $\partial D_{1}\left(\varepsilon^{\prime}\right) \times D_{2}(r) \cup A_{1} \times \partial D_{2}(r)$; совпадает со стандартной проекцией $C(L) \rightarrow L$ на $C(L) \cap T$, заданной конической структурой; и постоянна на каждом $H_{1, j}, j \geqslant 2$. Такая проекция (такое расслоение) задается потоком, определяемым некоторым векторным полем на $T$, которое является касательным к $C(L)$, ко всем $H_{1, j}, j \geqslant 2$, и к $A_{1} \times \partial D_{2}(r)$ и получено поднятием инвариантного при вращении и нигде не обращающегося в нуль радиального векторного поля на $A_{1}$. Образ $\operatorname{pr}^{\prime}\left(C_{-}\right)$, где $C_{-}=C(L) \cap \mathrm{pr}_{1}^{-1} I_{-}$, состоит из $l$ точек, которые обозначим через $w_{1}, \ldots, w_{l}$. Пусть $W$ - элемент из $\pi_{1}\left(T \backslash C(L),\left(-\varepsilon^{\prime}, w_{0}\right)\right)$, совпадающий с постоянным сечением $w=w_{0}$ над $\partial D_{1}\left(\varepsilon^{\prime}\right)$. Если $x=\operatorname{pr}_{*}^{\prime}(y)$, где $y \in \pi_{1}\left(T \backslash C(L),\left(-\varepsilon^{\prime}, w_{0}\right)\right)$ представим петлей, лежащей над $I_{-}$, то образ $x^{b}$ элемента $x$ при действии $b_{1}$ определяется равенствами $x^{b}=\operatorname{pr}_{*}^{\prime}\left(y^{b}\right), y W=W y^{b}$.

Теперь рассмотрим петлю $\bar{y}_{j}, l+1 \leqslant j \leqslant m$, которая начинается в $\left(-\varepsilon^{\prime}, w_{0}\right)$, спускается в $\left(-\varepsilon^{\prime}, w_{j}\right)$, не входя в $D_{2}\left(r^{\prime}\right)$, идет вдоль $H_{1, j-l+1}$ над $I_{-}$, возвращается вдоль $H_{2, j-l+1}$ и поднимается вверх в $\left(-\varepsilon^{\prime}, w_{0}\right)$, снова не входя в $D_{2}\left(r^{\prime}\right)$. Вращая $I_{-}$, получаем тор, для которого $\bar{y}_{j}$ является меридианом, а $W$ - параллелью. Следовательно, элемент $y_{j}$, представленный петлей $\bar{y}_{j}$, и элемент $W$ коммутируют, поэтому $x_{j}=\mathrm{pr}_{*}^{\prime} y_{j}$ является инвариантным элементом при действии $b_{1}$. Поскольку элемент $b_{1}$ нерасщепляем, то для любого $j$ существует изотопия диска $D_{2}(r) \backslash\left\{w_{1}, \ldots, w_{l}\right\}$, тождественная на границе, которая преобразует $\operatorname{pr}^{\prime} \bar{y}_{j}$ в некотую петлю, лежащую вне $D_{2}\left(r^{\prime}\right)$.

Наконец, чтобы преобразовать все $H_{1, j}$ в $H_{2, j}$ над $I_{-}$с помощью $H$-изотопии, тождественной на $\partial D \cup C(L)$, применим следуюший индуктивный процесс. На первом шаге с помощью $H$-изотопии, тождественной на $\partial D$ и $C(L)$, преобразуем $\mathrm{pr}^{\prime} \bar{y}_{2}$ в некоторую петлю, лежащую вне $D_{2}\left(r^{\prime}\right)$, а затем с помощью скручива- 
юще-раскручивающей изотопии преобразуем ее в постоянную петлю, т.е. отождествим $H_{2,2}$ с $H_{1,2}$ над $I_{-}$. Предположим, что мы уже получили $H_{1, j}=H_{2, j}$ над $I_{-}$для всех $j \leqslant i$, где $i<m-l+1$. Рассмотрим элемент, реализуемый в $\pi_{1}\left(D_{2}(r) \backslash\left\{w_{1}, \ldots, w_{l}, \ldots, w_{l+i-1}\right\}\right)$ петлей $\mathrm{pr}^{\prime} \bar{y}_{i+1}$. С помощью тех же аргументов, что и вьше, можно показать, что эта петля тоже инвариантна при действии $b_{1}$. Поэтому из леммы 3.2 следует, что эта петля является произведением элементов, соответствующих петле $\partial D_{2}\left(\varepsilon^{\prime}\right)$ и петлям вокруг $w_{j}, j>l$, не входящим внутрь диска $D_{2}\left(\varepsilon^{\prime}\right)$. Таким образом, снова, как и выше, можно применить $H$-изотопию, реализующую гомотопию петли $\mathrm{pr}^{\prime} \bar{y}_{i+1}$ в некоторую петлю, лежащую вне $D_{2}\left(r^{\prime}\right)$, а затем в результате скручивающе-раскручивающей $H$-изотопии преобразовать ее в постоянную петлю.

СлеДСТВИЕ 3.1. Любъе две кривъе Гурвица $\bar{H}_{1}, \bar{H}_{2} \subset \Sigma_{N}$ с особенностями нерасщепляемого типа изотопньи, если $\beta_{S}\left(\operatorname{bmt}\left(\bar{H}_{1}\right)\right)=\beta_{S}\left(\operatorname{bmt}\left(\bar{H}_{2}\right)\right)$.

ДоКАЗАТЕЛЬСТВО. С помощью изотопии можно слегка подвинуть точки, принадлежашие $Z(\bar{H})$, и получить $Z$-обшие кривые Гурвица, имеющие брейд-монодромный тип bmt $=\beta_{S}(\mathrm{bmt}(\bar{H}))$. Теперь доказательство следует из теоремы 3.2.

СЛЕДСТВИЕ 3.2. Z-общие кривые Гурвица с особенностями вида $w^{k}=z^{n}$ являются $H$-изотопными тогда и только тогда, когда они имеют один $u$ тот же тип разложения брәйд-монодромии на множители.

ДокАЗАТЕЛЬСТво следует из леммы 3.3.

В [11] доказано, что в случае $Z$-обших каспидальных кривых Гурвища можно сделать $H$-изотопию гладкой. Следуюшая теорема является обобщением этого результата на случай не $Z$-общих каспидальных кривых.

ТЕОРема 3.3. Каспидальные кривые Гурвица $\bar{H}_{1}, \bar{H}_{2} \subset \Sigma_{N}$ гладко H-изотопны тогда и только тогда, когда $\bar{H}_{1}$ и $\bar{H}_{2}$ имеют один и тот же тип разложения брәйд-монодромии на множители.

ДокАЗАТЕльство. Приведем здесь набросок доказательства. Стандартная форма брэйд-монодромии ростка каспидальной кривой над точкой, имеющего особенности типов $w^{2}=z^{n_{i}}$, определяется набором экспонент $\left\{n_{1}, \ldots, n_{t}\right\}$ и имеет следующий вид:

$$
\left(a_{1}^{n_{1}}, \mathbf{1}_{\varnothing}\right) \cdot\left(a_{3}^{n_{2}}, \mathbf{1}_{\varnothing}\right) \cdot \ldots \cdot\left(a_{2 t-1}^{n_{t}}, \mathbf{1}_{\varnothing}\right)
$$

где $2 t \leqslant m$.

Несложно показать (мы опускаем доказательство), что централизатор $C(b) \subset$ $B_{m}$ элемента

$$
b=a_{1}^{n_{1}} a_{3}^{n_{2}} \ldots a_{2 t-1}^{n_{t}}
$$

порождается элементами $a_{1}, a_{3}, \ldots, a_{2 t-1}, a_{2 t+1}, a_{2 t+2}, \ldots, a_{m-1}$, дополненными элементами $c_{i}, 1 \leqslant i \leqslant t$, и $d_{i, l}, i \neq l, 1 \leqslant i, l \leqslant t$, где

$$
\begin{aligned}
c_{i}= & a_{2 t} \ldots a_{2 i+1}\left(a_{2 i} a_{2 i-1}^{2} a_{2 i}\right) a_{2 i+1}^{-1} \ldots a_{2 t}^{-1} \\
d_{i, l}= & \left(a_{2 l} a_{2 l-1}\right) \ldots\left(a_{2 i-2} a_{2 i-3}\right)\left(a_{2 i} a_{2 i-1} a_{2 i+1} a_{2 i}\right)^{2} \\
& \times\left(a_{2 i-2} a_{2 i-3}\right)^{-1} \ldots\left(a_{2 l} a_{2 l-1}\right)^{-1}
\end{aligned}
$$


если $l<i$, и

$$
\begin{aligned}
d_{i, l}= & \left(a_{2 l-2} a_{2 l-1}\right) \ldots\left(a_{2 i+2} a_{2 i+1}\right)\left(a_{2 i} a_{2 i+1} a_{2 i-1} a_{2 i}\right)^{2} \\
& \times\left(a_{2 i} a_{2 i+1}\right)^{-1} \ldots\left(a_{2 l-2} a_{2 l-1}\right)^{-1}
\end{aligned}
$$

если $l>i$.

Теперь, как и в доказательстве теоремы 3.2, с помощью $H$-изотопии отождествляем $\bar{H}_{1}$ и $\bar{H}_{2}$ над дополнением к объединению малых дисков с центрами в точках из $\operatorname{pr}\left(Z\left(\bar{H}_{1}\right)\right)$. В каждом таком диске $D_{1}(\varepsilon)$ выбираем меньший диск $D_{1}\left(\varepsilon^{\prime}\right)$, над которым также можно отождествить $H_{1}$ и $H_{2}$ с одним и тем же несвязным объединением нескольких сечений и нескольких особенностей, заданных уравнениями $\left(w-w_{i}\right)^{2}=z^{n_{i}}$. После этого выбираем тривиализацию проекции pr над $D_{1}(\varepsilon) \backslash D_{1}\left(\varepsilon^{\prime}\right)$, относительно которой след $H_{1}$ кривой Гурвица $\bar{H}_{1}$ является радиально постоянным. Напомним, что $H_{2}$ и $H_{1}$ совпадают над границей кольца $D_{1}(\varepsilon) \backslash D_{1}\left(\varepsilon^{\prime}\right)$. Поскольку $H_{1}$ и $H_{2}$ имеют одну и ту же tbmf-форму

$$
\tilde{b}=\left(a_{1}^{n_{1}}, \mathbf{1}_{\varnothing}\right) \cdot\left(a_{3}^{n_{2}}, \mathbf{1}_{\varnothing}\right) \cdot \ldots \cdot\left(a_{2 t-1}^{n_{t}}, \mathbf{1}_{\varnothing}\right)
$$

то коса $g=\hat{r} \cap H_{2} \in B_{m}$, где $\hat{r}=\left\{z \in D_{1}(\varepsilon) \mid z \in \mathbb{R}, \varepsilon^{\prime} \leqslant z \leqslant \varepsilon\right\}$, коммутирует с

$$
b=a_{1}^{n_{1}} a_{3}^{n_{2}} \ldots a_{2 t-1}^{n_{t}} .
$$

Исходя из описания ценрализатора $C(b)$ элемента $b$, элемент $g \in C(b)$ можно записать в виде слова $\hat{g}$ в алфавите, состоящем из образующих группы $C(b)$ и обратных к ним элементам. Первую букву в этом слове можно удалить с помощью одной из следующих скручивающе-раскручивающих изотопий: каждую из букв $c_{i}$ и $a_{l}, l \geqslant 2 t+1$ (и обратных к ним), можно удалить скручивающе-раскручивающей изотопией, использованной в доказательстве теоремы $3.2 ; a_{2 i-1}$ при $1 \leqslant i \leqslant t$ можно удалить скручивающе-раскручивающей изотопией, описанной в [11], и каждое $d_{i, l}$ может быть удалено с помощью “композищии” этих двух типов изотопий. В результате последовательности таких изотопий связные компоненты следа кривой $H_{2}$ уже не будут зацеплены со связными компонентами следа кривой $H_{1}$, т.е. мы получим $g=\mathbf{1}$. После этого конец доказательства совпадает с соответствуюшей частью доказательства теоремы 3.2 .

3.3. Почти алгебраические кривые. Согласно теореме 2.1 для любого элемента $b \in \mathscr{P}$ сушествуют число $N \in \mathbb{N}$ и алгебраическая кривая $\bar{C} \subset \Sigma_{N}$, не пересекающаяся с $E_{N}$ (и пересекающая трансверсально $F_{\infty}$ ) такая, что ее афофинная часть $C \subset \Sigma_{N} \backslash\left(E_{N} \cup F_{\infty}\right)$ имеет над $D_{1}(1)$ разложение брэйд-монодромии на множители, равное $b$. Для $b \in \mathscr{P}$ минимальное число $N=N(b)$ такое, что $b$ является разложением на множители брэйд-монодромии некоторой алгебраической кривой $\bar{C} \in \mathbb{P} H^{0}\left(\Sigma_{N}, \mathscr{O}_{\Sigma_{N}}\left(m E_{N}+m N F\right)\right)$ над некоторым диском $D_{1}(r) \subset \mathbb{P}^{1}$ радиуса $r>0$, называется реализуемой степенью элемента $b$. Если $\beta(b)=\Delta^{2 k}$, то число

$$
d(b)=N(b)-k
$$

называется недостаточностью элемента $b$. 
ОПРЕДЕЛЕНИЕ 3.4. Кривая Гурвища $\bar{H} \subset \Sigma_{N}$ называется почти алгебраической кривой, если она совпадает с алгебраической кривой $C$ над некоторым диском $D_{1}(r)$ и является объединением $m$ попарно непересекающихся сечений $H_{\infty, 1}, \ldots$ $\ldots, H_{\infty, m}$ проекции рr над $\mathbb{P}^{1} \backslash D_{1}(r)$.

Следующая теорема является непосредственным следствием теорем 2.1, 3.2, 3.3.

Teоpema 3.4. (i) Для любого разложения $b \in \mathscr{P}$ такого, что $\beta(b)=\Delta^{2 N}$, найдется такая почти алгебрачческая кривая Гурвица $\bar{H} \subset \Sigma_{N}$, что разложение на множители ее брәйд-монодромии $b(\bar{H})$ равно $b$.

(ii) Каждая Z-общая кривая Гурвича $\bar{H} \subset \Sigma_{N}$ с особенностями типа $w^{k}=z^{n}$ (в общем случае, с особенностями нерасщепляемого типа) является H-изотопной некоторой почти алгебраической кривой. В случае каспидальной кривой Гурвица эта изотопия может быть выбрана гладкой.

Разложение брэйд-монодромии на множители $b(\bar{H})=b_{1} \cdot \ldots \cdot b_{n} \in \mathscr{P}$ для общей обыкновенной каспидальной кривой Гурвица $\bar{H} \subset \Sigma_{N}$ имеет следующий вид:

$$
b(\bar{H})=\prod_{i=1}^{n}\left(q_{i} a_{1}^{r_{i}} q_{i}^{-1}\right) \in \mathscr{P},
$$

где $r_{i}$ зависит от типа особенности $s_{i} \in Z: r_{i}=3$, если $s_{i}$ является каспом (т.е. $\bar{H}$ локально в точке $s_{i}$ задается уравнением $\left.y_{i}^{2}=x_{i}^{3}\right), r_{i}=2$, если $s_{i}$ является нодом (т.е. задается уравнением $y_{i}^{2}=x_{i}^{2}$ ), и $r_{i}=1$, если $s_{i}$ является точкой касания (т.е. задается уравнением $y_{i}^{2}=x_{i}$ ).

ПреДЛОЖЕНИЕ 3.1. Существует бесконечная последовательность $\bar{H}_{i} \subset$ $\Sigma_{1}, \quad i \in \mathbb{N}$, общих обыкновенных каспидальных почти алгебраических кривых степени 54 с 378 каспами и 756 нодами и с попарно различными типами разложений брәйд-монодромий на множители. В частности, все они не H-изотопны друг другу, почти все из них не изотопны алгебраическим каспидальным кривым и, более того,

$$
\lim _{i \rightarrow \infty} d\left(b\left(\bar{H}_{i}\right)\right)=\infty .
$$

ДокАЗАТЕльСТво. Существование последовательности $\bar{H}_{i}$, удовлетворяющей первой части утверждения, следует из теорем 2.1 и 3.4 , примененных к найденной Б. Мойшезоном (см. [17, теорема 1$])$ последовательности попарно различных типов каспидальных разложений элемента $\Delta_{54}^{2}$ на множители.

Зафиксируем $N$ и определим пространство $B$ как пространство алгебраических кривых

$$
\bar{C} \in \mathbb{P}=\mathbb{P} H^{0}\left(\Sigma_{N}, \mathscr{O}_{\Sigma_{N}}\left(54 E_{N}+54 N F\right)\right),
$$

не содержащих сечение $E_{N}$ в качестве компоненты, не имеющих критических точек над границей диска $D_{1}(1) \subset \mathbb{P}^{1}$ и имеюших $54(54-1)$ критических значений, лежащих в $D_{1}(1)$ (считаемых с кратностями); среди них 378 критических значений, соответствующих каспам, и 756 значений, соответствуюших нодам, и все остальные критичекие точки соответствуют простым касательным. Чтобы доказать, что $\lim _{i \rightarrow \infty} d\left(b\left(\bar{H}_{i}\right)\right)=\infty$, достаточно показать, что $B$ состоит из конечного числа компонент. Отметим, что $B$ содержится в пространстве $\mathscr{M}$ всех кривых в 
$\mathbb{P}$, имеюших по крайней мере 378 каспов и 756 нодов, и это пространство является квазипроективньгм многообразием.

Как и в доказательстве леммы 2.2 , рассмотрим отображение

$$
\mathscr{R}: \mathbb{P} \rightarrow \operatorname{Sym}_{54(54-1) N} \mathbb{P}^{1} \simeq \mathbb{P}^{54(54-1) N},
$$

которое определено в каждой точке пространства $B$, и $\mathscr{R}(B)$ содержится в открытом множестве

$$
\begin{aligned}
V=\left\{\left(z_{1}, \ldots, z_{54(54-1) N}\right)|| z_{i} \mid<1 \text { для } i \leqslant 54(54-1)\right. \\
\text { и } \left.\left|z_{i}\right|>1 \text { для } i>54(54-1)\right\},
\end{aligned}
$$

так что $B \subset \mathscr{R}^{-1}(V) \cap \mathscr{M}$. Множество $\mathscr{R}^{-1}(V) \cap \mathscr{M}$ является полуалгебраическим. Рассмотрим квазипроективную стратификацию пространства $\mathscr{M}$ на страты, соответствуюшие эквисингулярным кривым. Пересекая ее с $\mathscr{R}^{-1}(V)$, получаем конечную (полуалгебраическую) стратификацию множества $\mathscr{R}^{-1}(V)$. Пересечение пространства $B$ с каждым стратом этой стратификации является одновременно открытым и замкнутым множеством в рассматриваемом страте, следовательно, $B$ имеет только конечное число связных компонент.

ПроБлЕмА. Верно ли, что для любой нодальной почти алгебраической кривой $\bar{H} \subset \Sigma_{N}$ ее недостаточность $d(b(\bar{H}))$ равна нулю, т.е. $\bar{H}$ будет $H$-изотопна некоторой алгебраической нодальной кривой $\bar{C} \in \Sigma_{N}$ ?

\section{4. Несколько замечаний. Пусть}

$$
b(\bar{H})=\prod_{i=1}^{n} \lambda\left(q_{i}\right)\left(b_{i}\right) \in \mathscr{P}
$$

- разложение брэйд-монодромии кривой Гурвища $\bar{H} \subset \Sigma_{N}, \operatorname{deg} \bar{H}=m$, на множители, где каждый множитель $b_{i}$ является стандартной формой брэйд-монодромии алгебраической кривой над точкой. Каждый множитель $b_{i}$ раскладывается в произведение:

$$
b_{i}=b_{i, 1} \cdot \ldots \cdot b_{i, t_{i}},
$$

где $b_{i, 1} \in B_{k_{i, 1}, 0}^{+} \subset B_{m}$ и $B_{k_{i, 1}, 0}^{+}$- полугруппа в $B_{m}$, порожденная элементами $a_{1}, \ldots, a_{k_{i, 1}-1} ; b_{i, 2} \in B_{k_{i, 2}, k_{i, 1}}^{+}$и $B_{k_{i, 2}, k_{i, 1}}^{+}$порождается $a_{k_{i, 1}+1}, \ldots, a_{k_{i, 1}+k_{i, 2}-1}$; $\ldots ; b_{i, t_{i}} \in B_{k_{i, t_{i}}, k_{i, 1}+\cdots+k_{i, t_{i}-1}}^{+}$и $B_{k_{i, t_{i}}, k_{i, 1}+\cdots+k_{i, t_{i}-1}}^{+}$порождается элементами $a_{k_{i, 1}+\cdots+k_{i, t_{i}-1}+1}, \ldots, a_{k_{i, 1}+\cdots+k_{i, t_{i}}-1}$. Напомним, что $k_{i, j} \geqslant 2$ для $1 \leqslant j \leqslant t_{i}$.

С помощью этого разложения на множители можно описать гомотопический тип дополнения $\Sigma_{N} \backslash\left(\bar{H} \cup E_{N} \cup F_{\infty}\right)$ (ср. с [13]). Именно это дополнение имеет гомотопический тип двумерного комплекса Кэли представления (ван Кампена-Зариского) фундаментальной групшы

$$
\pi_{1}\left(\Sigma_{N} \backslash\left(\bar{H} \cup E_{N} \cup F_{\infty}\right)\right)=\left\langle x_{1}, \ldots, x_{m}: R_{i, j, k}\right\rangle ;
$$

в терминах канонического действия группы кос $B_{m}$ на свободной группе, порожденной $x_{1}, \ldots, x_{m}$, определяюшие соотношения $R_{i, j, k} \quad\left(i=1, \ldots, n, j=1, \ldots, t_{i}\right.$, $\left.k_{i, 1}+\cdots+k_{i, j-1}+1 \leqslant k \leqslant k_{i, 1}+\cdots+k_{i, j}-1\right)$ имеют вид

$$
q_{i}^{-1}\left(b_{i, j}\left(x_{k}\right)\right)=q_{i}^{-1}\left(x_{k}\right) \text {. }
$$


Отметим также, что, используя аргументы, приведенные в [1], можно показать, что тип разложения на множители брэйд-монодромии топологической кривой Гурвица в $\Sigma_{N}$ не меняется при действии некоторых специальных объемлющих гомеоморфизмов. Именно, свяжем с каждой топологической кривой Гурвица $\bar{H}$ стратифицированное объединение $\widetilde{H}=\bar{H} \cup\left(\bigcup_{s \in Z} F_{\operatorname{pr}(s)}\right) \cup F_{\infty} \subset \Sigma_{N}$ (и назовем его оснащенной кривой); две топологические кривые Гурвица $\bar{H}_{1}, \bar{H}_{2}$ имеют один и тот же тип разложения брэйд-монодромии на множители, если (и только если) существует гомеоморфизм ориентированных пар $h:\left(\Sigma_{N}, \widetilde{H}_{1}\right) \rightarrow\left(\Sigma_{N}, \widetilde{H}_{2}\right)$, который отображает $\bar{H}_{1}$ в $\bar{H}_{2}$ и оставляет инвариантным $F_{\infty}$.

Условие, что $h$ сохраняет ориентации компонент топологических кривых Гурвица, является сушественным. Это следует из примеров [10] неприводимых (обыкновенных) каспидальных алгебраических кривых $C_{1}, C_{2} \subset \Sigma_{1}$, имеющих различные типы разложений брэйд-монодромии на множители, но для которых существует диффеоморфизм $c:\left(\Sigma_{1}, \widetilde{C}_{1}\right) \rightarrow\left(\Sigma_{1}, \widetilde{C}_{2}\right)$, не сохраняюший ориентацию компонент оснашенных кривых (в этих примерах $c$ является комплексным сопряжением).

\section{§4. Разложения на множетели брэйд-монодромий симплектических поверхностей}

В этом параграфе мы рассматриваем особые симплектические поверхности в $\mathbb{C P}^{2}$. Как известно, $\mathbb{C P}^{2}$ обладает единственной с точностью до симплектоморфизмов и умножения на постоянный множитель симплектической структурой. С другой стороны, насколько известно авторам, вопрос о ее единственности с точностью до изотопии и умножения на постоянный множитель является открытым. Более того, перемасштабирование симплектической структуры является важной составляющей доказательства предложения 4.2, поэтому рассмотрим изотопические классы симплектических кривых.

Далее изучаем поверхности только с изолированными особенностями. Поэтому под особой симплектической поверхностью $C \subset \mathbb{C P}^{2}$ подразумеваем тройку $(C, J, \omega)$, где $C=f(\mathfrak{S})$ - образ почти всюду инъективного $J$-голоморфного отображения $f: \mathfrak{S} \rightarrow \mathbb{C P}^{2}$ замкнутой римановой поверхности $\mathfrak{S}, J$ - некоторая почти комплексная структура, определенная в некоторой окрестности поверхности $C$ и являющаяся ручной относительно заданной симплектической структуры $\omega$ на $\mathbb{C P}^{2}$. Говорят, что две особые симплектические поверхности $C_{0}=$ $f_{0}(\mathfrak{S}), C_{1}=f_{1}(\mathfrak{S})$ являются слабо симплектически (әладко) изотопнылми, если $\left(C_{0}, J_{0}^{\text {loc }}, \omega_{0}\right),\left(C_{1}, J_{1}^{\text {loc }}, \omega_{1}\right)$ могут быть включены в непрерьвное (соответственно, гладкое) семейство ручных почти комплексных и симплектических структур $J_{t}^{\text {loc }}$, $\omega_{t}$ и $J_{t}^{\text {loc }}$-голоморфных отображений $f_{t}: \mathfrak{S} \times[0,1] \rightarrow \mathbb{C P}^{2}$ такие, что отображения $\phi_{t}: C_{0} \rightarrow C_{t}$, заданные формулой $f_{0}(s) \mapsto f_{t}(s), s \in \mathfrak{S}$, являются хорошо определенными гомеоморфизмами для всех $t \in[0,1]$.

Аналогичные определения применимы к симплектическим поверхностям на любом многообразии. В случае, когда симплектическая структура не изменяется, говорят о симплектической изотопии. В частности, две особые симплектические поверхности симплектически изотопны, если существует симплектическая диффеотопия, преобразуюшая одну поверхность в другую.

ЗАмЕчАнИЕ 4.1. В случае произвольных симплектических многобразий естественно ожидать, что классы симплектически изотопных поверхностей и классы 
слабо симплектически изотопных поверхностей не совпадают в общем случае. Однако эти классы совпадают в случае симплектических структур на $\mathbb{C P}^{2}$. Действительно, тогда любая слабо симплектическая изотопия может быть перемасштабирована $\omega_{t} \mapsto \omega_{t}^{\prime}=\lambda_{t} \omega_{t}, \lambda_{t} \in \mathbb{R}_{+}$(не изменяя $J_{t}$ ), в изотопию форм $\omega_{t}^{\prime}$, не меняющую класс когомологий этих форм. После этого остается применить теорему Мозера и получить диффеотопию, преобразующую $\omega_{t}^{\prime}$ в постоянную форму; та же самая диффеотопия затем применяется к $f_{t}$ и $J_{t}^{\text {loc }}$. Поэтому, когда требуется доказать, что некоторые симплектические поверхности в $\mathbb{C P}^{2}$ симплектически изотопны, достаточно проверить, что они слабо симплектически изотопны. Ниже будем следовать этой стратегии.

ЗАмЕчАнИЕ 4.2. Пространство почти комплексных структур на конечномерном вещественном векторном пространстве, ручных относительно заданной симплектической формы, является стягиваемым (см. [9]). Отсюда следует, что любая $\omega$-ручная почти комплексная структура $J^{\text {loc }}$, определенная на открытом подмножестве $U$ симплектического многообразия $(V, \omega)$, может быть продолжена с меньшей окрестности $U_{0} \subset U$ до $\omega$-ручной почти комплексной структуры $J$ на всем $V$. Более того, по той же причине, если $U=U_{t}, J^{\mathrm{loc}}=J_{t}^{\mathrm{loc}}, \omega=\omega_{t}$ гладко зависят от одного или нескольких параметров $t$, это продолжение с $U_{0}=U_{0, t}$ на $\mathbb{C P}^{2}$ может быть выбрано гладко зависяшим от $t$. Следовательно, мы получим те же самые понятия, если в данных выше определениях заменим заданные локально $J$-структуры на ручные почти комплексные структуры, определенные на всем $\mathbb{C P}^{2}$. Выбор определений, который мы сделали, мотивирован упрошением некоторых доказательств. Отметим также, что приведенные вьше свойства продолжения остаются верными, если дополнительно ограничиться рассмотрением случая почти комплесных структур, для которых данная симплектическая поверхность является $J$-голоморфной. В частности, в определении слабой симплектической изотопии поверхностей достаточно требовать, чтобы $J_{t}^{\text {loc } б ы л и ~ з а д а н ы ~ т о л ь к о ~ в б л и з и ~ о с о-~}$ бых точек поверхностей $C_{t}$.

Как известно (см. [21], [15]), особенности псевдоголоморфных кривых на почти комплексных четырехмерных многообразиях эквивалентны с точностью до $C^{1}-$ замены координат особенностям настоящих комплексных кривых, лежащих на комплексных поверхностях. В случае, когда особая симплектическая поверхность $C$ задана вместе с симплектической структурой $\omega$ и $\omega$-ручной почти комплексной структурой $J^{\text {loc }}$ в некоторой окрестности $U$ поверхности $C$ в $\mathbb{C P}^{2}$, можно продолжить $J^{\text {loc }}$ с меньшей окрестности $U_{0} \subset U$ до $\omega$-ручной почти комплексной структуры $J$ на всем $\mathbb{C P}^{2}$, а после этого рассмотреть общий пучок $L$, составленный из $J$-прямых (см. [9] и [22]), и определить брэйд-монодромный тип $\operatorname{bmt}(C, \omega, J, L)$ аналогично тому, как он был определен в п. 2.3 в алгебраическом случае.

Отметим, что любой элемент $b \in \mathscr{P}$ такой, что $\alpha(b)=\Delta^{2}$ (и только такие элементы), может быть реализован как разложение на множители брэйд-монодромии некоторой симплектической поверхности относительно некоторого (не обязательно общего) пучка. Такая реализация может быть получена из почти алгебраической кривой с таким же разложением брэйд-монодромии (см. теорему 3.4) после перемасштабирования стандартного пучка прямых, относительно которого эта почти алгебраическая кривая задана.

ПРЕДЛОЖЕНИЕ 4.1. Тип брэйд-монодромии $\operatorname{bmt}(C, \omega, J, L)$ относительно общего пучка $L$ зависит только от класса симплектически изотопных по- 
верхностей, которому принадлежит $C$. (В частности, он не зависит от продолжения структуры $J^{\text {loc }}$, определенной локально, на все $\mathbb{C P}^{2}$ до почти комплексной структуры $J$.

ДокАЗАТЕЛЬСтво. Пусть $C_{0}, C_{1}$ - слабо симплектически изотопные симплек-

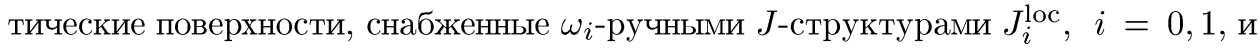
пусть $J_{0}, J_{1}$ - продолжения этих структур, а $L_{0}, L_{1}$ - обшие пучки такие же, как и в определениях особых симплектических поверхностей и соответствуюших им разложений брэйд-монодромий на множители. Выберем слабую симплектическую изотопию $\left(C_{t}, \omega_{t}, J_{t}^{\text {loc }}\right)$ и продолжим $J_{t}^{\text {loc }}$ до изотопии структур $J_{t}$ на все $\mathbb{C P}^{2}$. Для каждого $t$ пространство $\left(\mathbb{C P}_{t}^{2}\right)^{*}$, составленное из $J_{t}$-прямых, диффеоморфно $\mathbb{C P}^{2}$ и снабжено канонической двойственной $E$-структурой (см. [22]). Касательные $J_{t}$-прямые к $C_{t}$ составляют двойственную $E$-кривую $C_{t}^{*}$, которая имеет конечное число особых точек (соответствующих двойным касательным или касательным с более сильным касанием), и двойственная к $C_{t}^{*}$ поверхность совпадает с $C_{t}$. Поэтому пучок является общим, если его центр не принадлежит ни $C_{t}$, ни конечному числу $J_{t}$-прямых, двойственных особым точкам кривой $C_{t}^{*}$. Следовательно, найдется путь $L_{t}$, состоящий из общих пучков и соединяющий $L_{0}$ с $L_{1}$. Это завершает доказательство, так как ясно, что разложения на множители брэйд-монодромий поверхностей $C_{t}$ относительно пучков $L_{t}$ не зависят от $t$.

Напомним, что тип разложения на множители брэйд-монодромии $m$ комплексных прямых в $\mathbb{C P}^{2}$, находящихся в общем положении, совпадает с элементом $\tilde{\delta}_{m}^{2}$ (см., например, [18]), который был определен в п. 1.4.

СЛЕДСТВИЕ 4.1. Тип разложения на множители брәйд-монодромии общего семейства из $m$ псевдопрямых равен $\tilde{\delta}_{m}^{2}$.

ДокАЗАТЕЛЬСтво. В соответствии с [2] каждое общее семейство, состояшее из $m$ псевдопрямых, симплектически изотопно $m$ комплексным прямым в общем положении.

СЛЕДСТВИЕ 4.2. Существует бесконечно много плоских обыкновенных каспидальных симплектических поверхностей одной и той же степени и с совпадающими числом каспов и числом нодов, но попарно симплектически не изотопных.

ДОКАЗАТЕЛЬСТВо следует из предложений 3.1, 4.1.

ЗАмЕчАнИЕ 4.3. Обратное к предложению 4.1 утверждение также верно, если симплектическая изотопия заменена топологической и дополнительно предположено, что все особенности нерасщепляемого типа. Действительно, из [12] следует, что пучки, определенные двумя почти комплексными структурами, являющимися ручными для одной и той же симплектической структуры, или, более общо, ручными в непрерывном семействе симплектических структур, являются изотопньми. Отсюда и из теоремы 3.2 вытекает, что если брэйд-монодромные типы двух особых симплектических (относительно одной и той же симплектической структуры, либо относительно структур, принадлежащих одной связной компоненте) поверхностей с особыми точками нерасщепляемого типа совпадают, то поверхности топологически изотопны. Могут возникнуть проблемы при замене топологической изотопии на гладкую, так как в гладкой категории предполагается, что изотопия 
является объемлющей, тогда как наш выбор определения гладких симплектических изотопий не предполагает этого. Несомненно, объемлющую изотопию можно сгладить вне особых точек, но в общем случае ее нельзя сгладить в особых точках, так как они могут иметь модули даже относительно гладкой замены координат.

ЗАмЕчАНИЕ 4.4. В следствии 4.1 псевдопрямые можно заменить на кривые Гурвица, реализуюшие образуюшую группы $H_{2}\left(\mathbb{C P}^{2}\right)$ (напомним, что в соответствии с нашими определениями кривые Гурвица определены относительно стандартного пучка прямых), так как после перемасштабирования трансверсального пучка кривые Гурвица становятся $J$-кривыми, т.е. становятся псевдопрямыми. Как авторам сообшил В. Шевчишин, результат Барро (Barraud) может быть обобщен на нодальные симплектические кривые (без отрицательных нодов) рода $\leqslant 3$. Поэтому приведенные выше аргументы могут быть применены также и к этим криBЫМ.

Следуюшее предложение является частично обратным к предложению 4.1.

ПРЕДЛОЖЕНИЕ 4.2. Две симплектические относительно симплектической структурь Фубини-Штуди $\omega_{0}$ обькновенные каспидальные поверхности являются симплектически $C^{1}$-гладко изотопными в $\mathbb{C P}^{2}$ тогда и только тогда, когда они имеют один и тот же тип разложения брәйд-монодромии относительно общих пучков.

ДоКАЗАТЕльСТВо. Необходимость совпадения типов разложений на множители брэйд-монодромий следует из предложения 4.1.

Из замечания 4.1 следует, что для доказательства обратного утверждения достаточно найти слабую симплектическую изотопию.

Пусть $C_{0}$ - произвольная симплектическая поверхность с обыкновенными кас-

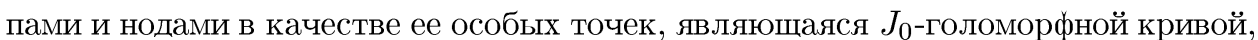
где $J_{0}$ - почти комплексная структура на $\mathbb{C P}^{2}$, совместимая с $\omega_{0}$. С помощью неперывной вариации структуры $J_{0}$ с носителем в некоторой окрестности точки $p$ преобразуем $J_{0}$ в интегрируемую структуру в более малой окрестности $U_{0}^{\prime}$. После этого рассмотрим общий пучок $L_{0}$ псевдопрямых с центром в $p_{0} \in U_{0}^{\prime}$. Выберем локальные координаты $x, y$ вблизи критических точек поверхности $C_{0}$ (под критuческими точками мы понимаем особые точки поверхности $C_{0}$ и точки касания $C_{0} \mathrm{c}$ прямыми из пучка $L_{0}$ ) так, что локально элементы $J_{0}$-пучка являются слоями проекции $(x, y) \mapsto x$, а $C_{0}$ задается уравнениями вида $y^{2}=x^{k}, k=1,2,3$, в зависимости от того, является ли эта точка точкой касания, нодом или каспом соответственно; во всех этих случаях направление $y=0$ может быть выбрано симплектическим. Это позволяет заменить $J_{0}$ на $\omega_{0}$-ручную почти комплексную структуру $J_{0}^{\prime}$, относительно которой выбранные выше координаты становятся $J$-голоморфиными (так что $J_{0}^{\prime}$ является интегрируемой вблизи критических точек и вблизи $p_{0}$ ), а $C_{0}$ и пучок $L_{0}$ остаются $J$-голоморфньми. Поскольку $J_{0}$ и $J_{0}^{\prime}$ являются $\omega_{0}$-ручными и поскольку $C_{0}$ является $J$-голоморфной кривой относительно обеих структур, они могут быть соединены гомотопией почти комплексных структур, оставляющих $C_{0}$ $J$-голоморфной кривой. Согласно предложению $4.1, \operatorname{bmt}\left(C_{0}\right)$ не меняется. Из $[14$, лемма 5.5BC] следует сушествование слабо симплектической изотопии, имеющей носитель в $U_{0}^{\prime}$, состоящей из непрерьвной вариации формы $\omega_{0}$ и заменяющей пару $\left(\omega_{0}, J_{0}^{\prime}\right)$ на стандартную (т.е. плоскую, келерову) в $U_{0} \subset U_{0}^{\prime}$ пару $\left(\omega_{0}^{\prime}, J_{0}^{\prime}\right)$. 
$\mathrm{C}$ другой стороны, согласно теореме 3.1 существует кривая Гурвища $C_{1} \subset \mathbb{C P}^{2}$, у которой тип брэйд-монодромии (относительно стандартного пучка обыкновенных прямых $\left.L_{1}\right) \operatorname{bmt}\left(C_{1}\right)$ совпадает c $\operatorname{bmt}\left(C_{0}\right)$ (напомним, что $\operatorname{bmt}\left(C_{0}\right)$ определен с помошью обшего пучка $J_{0}$-прямых). Перемасштабируя обший пучок $L_{1}$ и перемешая его центр в $p_{0}$, можно считать, что $C_{1}$ является $J_{1}$-голоморфной кривой, где $J_{1}$ - подходящая $\omega_{0}$-ручная почти комплексная структура, совпадаюшая со стандартной комплексной структурой в некоторой окрестности точки $p_{0}$ и в некоторых окрестностях критических точек кривой $C_{1}$.

Кроме того, как и выше, можно заменить $\left(\omega_{0}, J_{1}\right)$ на стандартную вблизи точки $p_{0}$ пару $\left(\omega_{1}^{\prime}, J_{1}^{\prime}\right), J_{1}^{\prime}=J_{1}$. Для доказательства предложения достаточно показать, что $C_{0}$ и $C_{1}$ являются слабо симплектически $C^{1}$-гладко изотопными в $\mathbb{C P}^{2}$.

Как и в доказательстве предложения 4.1 , выберем путь $\left(\omega_{t}^{\prime}, J_{t}^{\prime}\right)$ с $\omega_{t}^{\prime}$-ручными почти комплексными структурами $J_{t}^{\prime}$, интегрируемыми вблизи $p_{0}$, и рассмотрим семейство $J_{t}^{\prime}$-голоморфных пучков $J_{t}^{\prime}$-прямых, соединяюшее пучки $\left(J_{i}^{\prime}, L_{i}\right)$, $i=0,1$. С помощью непрерывной вариации сделаем почти комплексные структуры $J_{t}^{\prime}$ интегрируемыми вблизи критических точек. Теперь осталось построить изотопию, переводящую $C_{0}^{\prime}$ в $C_{1}$, и подправить ее так, чтобы она стала слабо симплектической изотопией. Построение изотопии осушествим в два шага.

На первом шаге с помощью диффеотопии пучков, голоморфной вблизи $p_{0}$ и вблизи критических точек, строим диффеотопию $C_{t}^{\prime}$ поверхности $C_{0}=C_{0}^{\prime}$. Это дает семейство поверхностей $C_{t}^{\prime}$, голоморфных вблизи критических точек, а также дает аналог $H$-изотопии: вне критических точек каждая поверхность $C_{t}^{\prime}$ пересекает псевдопрямые трансверсально с положительньм индексом пересечения; проекция каждой поверхности $C_{t}^{\prime}$ на базу соответствуюшего пучка является конечным разветвленным накрытием; вблизи критических точек поверхность является комплексно-аналитической кривой с уравнением $y^{2}=x^{k}, k=1,2,3$, в локальных аналитических координатах $x, y$, в которых проекция задана как $(x, y) \mapsto x$. Получающаяся в результате поверхность $C_{1}^{\prime}$ является кривой Гурвица в смысле определения 3.1, так как $L_{1}$ является пучком обыкновенных прямых. Очевидно, $\operatorname{bmt}\left(C_{1}^{\prime}\right)=\operatorname{bmt}\left(C_{0}\right)$.

На втором шаге аргументы, приведенные в [11], могут быть применены к двум кривым Гурвица $C_{1}^{\prime}$ и $C_{1}$, имеюшим один и тот же тип разложения на множители брэйд-монодромии относительно пучка $L_{1}$, чтобы построить $H$-изотопное семейство кривых Гурвища $H_{t}$, соединяюшее $H_{0}=C_{1}^{\prime}$ и $H_{1}=C_{1}$ и имеюшее все перечисленные выше свойства.

Чтобы построить слабо симплектическую изотопию с помощью объединенной построенной вьше изотопии $\left(H_{t}\right.$ следует за $\left.C_{t}^{\prime}\right)$, применим к семействам $\left(C_{t}^{\prime}, \omega_{t}^{\prime}\right)$ и $\left(H_{t}, \omega_{1}^{\prime}\right)$ следуюшее перемасштабирование форм $\omega_{t}^{\prime}$. Как и в начале доказательства, используем конструкцию из [14], чтобы непрерывной вариацией форм $\omega_{t}^{\prime}$ получить семейство пар $\left(\omega_{t}^{\prime}, J_{t}^{\prime}\right)$, совпадающих со стандартной плоской парой $(\Omega, i)$ в малом шаре $B(\delta), \delta>0$, с центром в $p_{0}$. После этого строим $\mathbb{C}^{1}$-расслоения $h_{t}: \mathbb{C P}^{2} \backslash\left\{p_{0}\right\} \rightarrow S^{2}$, которые имеют симплектические слои $h_{t}^{-1}(v), v \in S^{2}$, и которые совпадают со старыми $J_{t}^{\prime}$-пучками $\mathrm{pr}: \mathbb{C P}^{2} \backslash\left\{p_{0}\right\} \rightarrow S^{2}$ вне $B(\delta)$ и со стандартным пучком прямых в меньшем шаре $B\left(\delta^{\prime}\right)$. Как только такие расслоения построены, остается заменить $\omega_{t}^{\prime}$ следующим образом: вне шара $B\left(\delta^{\prime}\right)$ заменим на $\omega_{t}^{\prime}+N h_{t}^{*} \omega_{S^{2}}$, где $N \geqslant 0$ - достаточно большая константа и $\omega_{S^{2}}-$ форма объема на $S^{2}$; а внутри $B\left(\delta^{\prime}\right)$ заменим на $\Omega_{N}, \Omega_{N}=\omega_{t}^{\prime}+N h_{t}^{*} \omega_{S^{2}}=\Omega+N h^{*} \omega_{S^{2}}$, 
вблизи гранищы $\partial B\left(\delta^{\prime}\right)$ (через $h$ обозначаем расслоение на стандартные прямые). Такое семейство $\Omega_{N}$ найдено в $[14$, предложение $5.1 \mathrm{~B}]$. Чтобы получить недостающую слабую симплектическую изотопию между $\left(C_{0}^{\prime}, \omega_{0}^{\prime}\right)$ и $\left(C_{0}^{\prime}, \omega_{0}^{\prime}+N \mathrm{pr}^{*} \omega_{S^{2}}\right)$, а также между $\left(H_{1}, \omega_{1}^{\prime}+N \mathrm{pr}^{*} \omega_{S^{2}}\right)$ и $\left(H_{1}, \omega_{1}^{\prime}\right)$, достаточно проварьировать только симплектическую структуру: $\theta \mapsto \omega_{i}^{\prime}+\theta \mathrm{pr}^{*} \omega_{S^{2}}, i=1,2$.

Существование $h_{t}$ с перечисленными вьше свойствами можно доказать следующим образом. Занумеруем псевдопрямые, проходящие через $p_{0}$, точками сферы $S^{2}$, которую мы отождествляем с проективной прямой комплексных направлений в точке $p_{0}$. Затем, чтобы построить искомое $h_{t}$, фиксируем параметрическое представление псевдопрямой, проходящей через $p_{0}$, следующим образом. В качестве значения $\infty$ берем точку пересечения ее с некоторой выбранной псевдопрямой, не проходящей через $p_{0}$, а в качестве 0 берем точку $p_{0}$ и фиксируем производную параметризации в 0 (единичный касательный вектор $\xi$ в $\mathbb{C P}^{2}$ в точке $\left.p_{0}\right)$. С помощью этой параметризации $\phi_{\xi, t}: \mathbb{C P}^{1} \rightarrow \mathbb{C P}^{2}$ задаем слои расслоения $h_{t}$, заменяя $\phi_{\xi, t}$ в диске $0 \in D_{1}(\varepsilon) \subset \mathbb{C} \subset \mathbb{C P}^{1}$ на $(1-\delta(r)) \Xi+\delta(r) \phi_{\xi, t}: D_{1}(\varepsilon) \rightarrow B(\delta)$, где $\Xi-$ линейная часть параметризации $\phi_{\xi, t}$ в точке 0 и $\delta:[0, \varepsilon] \rightarrow[0,1]-$ некоторая функция, принимающая значения нуль вблизи точки 0 и единица вблизи $\varepsilon$. Полученные отображения $h_{t}$ имеют перечисленные выше свойства, если $\varepsilon$ выбрано достаточно малым и $r \delta^{\prime}(r)<1$ для любого $r \in[0, \varepsilon]$. (С помощью прямого вычисления в афрфинных координатах $z, w$ вблизи точки $p_{0}$, где $\operatorname{Re} \frac{\partial}{\partial z}=\xi$, можно проверить, что слой в направлении $\xi$ является симплектическим. Для этого необходимо записать форму $i d h \wedge \overline{d h} \wedge \Omega$ в данных координатах и использовать комплексную аналитичность частных производных по координатам $s$ уравнения $w=s z+\phi_{2}(s) z^{2}+\cdots$, которое определяет псевдопрямые, где $s$ - локальные координаты на $S^{2}$. Теперь можно использовать упомянутое выше уравнение, чтобы получить неявное уравнение для $\left.h: h=v-\delta(r)\left[\phi_{2}(h) z+\cdots\right], w=v z.\right)$

СЛЕДСТВИЕ 4.3. Нодальная симплектическая относительно симплектической структурь Фубини-Штуди поверхность является симплектически $C^{1}$-гладко изотопной алгебраической кривой тогда и только тогда, когда ее брэйд-монодромный тип bmt относительно общего пучка является частичной регенерацией некоторого әлемента из $S_{A_{1}}$ : $\mathrm{bmt}=r\left(z_{1}\right) \cdot z_{2}$, әде $r: S_{A_{1}} \rightarrow S_{A_{0}}-$ регенерачия (см. пример 1.1), $A_{0}$ - полное множество сопряжсенных образуюшей $a_{1} \in B_{m}, a A_{1}$ - полное множсество әлементов, сопряженных әлементу $a_{1}^{2}, u z_{1}, z_{2} \in S_{A_{1}}$.

ДокаЗАТЕльство. Во-первых, заметим, что $\alpha(\mathrm{bmt})=\Delta_{m}^{2}$, где $m$ - степень симплектической поверхности. Поэтому в соответствии с теоремой 1.2 имеем $z_{1} \cdot z_{2}=\tilde{\delta}_{m}^{2}$. Элемент, стоящий справа, является разложением на множители брэйд-монодромии $m$ прямых, находяшихся в общем положении. Можно сгладить соответствующие ноды этой алгебраической кривой, не деформируя остальные, что всегда возможно сделать, например, по теореме Брюзотти [5].

\section{Список литературы}

1. Artal E., Carmona J., Cogolludo J. I. Effective invariants of braid monodromy and topology of plane curves. AG/0105157.

2. Barraud J.-F. Nodal symplectic spheres in $\mathbb{C P}^{2}$ with positive self-intersection // Internat. Math. Res. Notices. 1999. № 9. P. 495-508. 
3. Ben-Itzhak T., Teicher M. Properties of Hurwitz equivalence in the braid group of order n. AG/0103194.

4. Brieskorn E., Knörrer H. Plane algebraic curves. Basel-Boston-Stuttgart: Birkhäuser Verlag, 1986.

5. Brusotti L. Sulla "piccola variazione" di una curva piana algebrica reali // Rend. Rom. Acc. Lincei. 1921. V. 30. № 5. P. 375-379.

6. Chisini $O$. Una suggestiva rapresentazione reale per le curve algebriche piane // Rend. Ist. Lombardo. II. 1933. V. 66. P. 1141-1155.

7. Chisini $O$. Courbes de diramation des plans multiples et tresses algébriques // Deuxième Colloque de Géométrie Algébrique. Paris: Masson \& Cie, 1952. P. 11-27.

8. Garside F. A. The braid group and other groups // Quart. J. Math. Oxford. 1969. V. 20. № 2. P. 235-254.

9. Gromov M. Pseudoholomorphic curves in symplectic manifolds // Invent. Math. 1985. V. 82. P. 307-347.

10. Kharlamov V. M., Kulikov Vik. S. Diffeomorphisms, isotopies and braid monodromy factorizations of plane cuspidal curves // C. R. Acad. Sci. Paris. Sér. I. Math. 2001. V. 333. P. $855-859$.

11. Куликов Вик. C., Тейчер М. Брэйд-монодромные разложения на множители и диффеоморфные типы // Изв. РАН. Сер. матем. 2000. Т. 64. № 2. С. 89-120.

12. Lalonde F., McDuff D. The classification of ruled symplectic 4-manifolds // Math. Research Letters. 1996. V. 3. P. 769-778.

13. Libgober A. On the homotopy type of the complement to plane algebraic curves // J. für die reine und angewandte Math. 1986. V. 367. P. 103-114.

14. McDuff D., Polterovich L. Symplectic packings and algebraic geometry // Inv. Math. 1994. V. 115. P. 405-429.

15. Micallef $M$. J., White $B$. The structure of branch points in minimal surfaces and in pseudoholomorphic curves // Ann. of Math. 1995. V. 141. № 2. P. 35-85.

16. Moishezon B. Stable branch curves and braid monodromies // Lecture Notes in Math. 1981. V. 862. P. 107-192.

17. Moishezon B. The arithmetic of braids and a statement of Chisini // Contemporary Math. 1994. V. 164. P. 151-175.

18. Moishezon B., Teicher $M$. Braid group technique in complex geometry. I: line arrangements in $\mathbb{C P}^{2} / /$ Contemporary Math. 1988. V. 78. P. 425-555.

19. Orevkov S. Yu. Realizability of a braid monodromy by an algebraic function in a disk // C. R. Acad. Sci. Paris. Sér. I. Math. 1998. V. 326. P. 867-872.

20. Rudolph L. Algebraic functions and closed braids // Topology. 1983. V. 22. P. 191-202.

21. Sikorav J.-C. Singularities of J-holomorphic curves // Math. Z. 1997. V. 226. P. 359-373.

22. Sikorav J.-C. Dual elliptic structures on $\mathbb{C P}^{2}$. math.SG/0008234.

23. Viro $O$. Gluing of plane real algebraic curves and constructions of curves of degrees 6 and 7 // Lecture Notes in Math. 1984. V. 1060. P. 187-200. 